\title{
ON THE TETRAHEDRALLY SYMMETRIC MONOPOLE
}

\author{
H.W. BRADEN AND V.Z. ENOLSKI
}

\begin{abstract}
We study $S U(2)$ BPS monopoles with spectral curves of the form $\eta^{3}+$ $\chi\left(\zeta^{6}+b \zeta^{3}-1\right)=0$. Previous work has has established a countable family of solutions to Hitchin's constraint that $L^{2}$ was trivial on such a curve. Here we establish that the only curves of this family that yield BPS monopoles correspond to tetrahedrally symmetric monopoles. We introduce several new techniques making use of a factorization theorem of Fay and Accola for theta functions, together with properties of the Humbert variety. The geometry leads us to a formulation purely in terms of elliptic functions. A more general conjecture than needed for the stated result is given.
\end{abstract}

\section{Contents}

1. Introduction

2. The Spectral Curve and its Constraints

2.1. Hitchin Data

2.2. The curve and its properties

3. Fay-Accola factorization 8

4. The Humbert variety 11

5. The Theta function Question

6. Discussion 17

Acknowledgements 18

Appendix A. Theta Functions 18

Appendix B. Proof of Proposition 17 19

Appendix C. Proof of Proposition 18

References 23

\section{INTRODUCTION}

For many years there has been considerable interest in magnetic monopoles, the topological soliton solutions of Yang-Mills-Higgs gauge theories in three space dimensions with particle-like properties. In particular BPS monopoles have been the focus of much research (see MS04 for a recent review). These monopoles satisfy a rather ubiquitous first order Bogomolny equation

$$
B_{i}=\frac{1}{2} \sum_{j, k=1}^{3} \epsilon_{i j k} F^{j k}=D_{i} \Phi
$$

(together with certain boundary conditions, the remnant of a limit in which the Higgs potential is removed). Here $F_{i j}$ is the field strength associated to a gauge field $A$, and $\Phi$ is the Higgs field. The Bogomolny equation may be viewed as a dimensional reduction of the four dimensional self-dual equations upon setting all functions independent of $x_{4}$ and 
identifying $\Phi=A_{4}$; they are also encountered in supersymmetric theories when requiring certain field configurations to preserve some fraction of supersymmetry. The study of BPS monopoles is intimately connected with integrable systems. Nahm gave a transform of the ADHM instanton construction to produce BPS monopoles [Nah82] and the resulting Nahm's equations have Lax form with corresponding spectral curve $\hat{\mathcal{C}}$. Hitchin gave a twistorial description of this curve Hit82: just as Ward's twistor transform relates instanton solutions in $\mathbb{R}^{4}$ to certain holomorphic vector bundles over the twistor space $\mathbb{C P}^{3}$, Hitchin showed that the dimensional reduction leading to BPS monopoles could be made at the twistor level as well and also obtained the same curve lying in mini-twistor space, $\hat{\mathcal{C}} \subset \mathrm{TP}{ }^{1}$. Subject to certain nonsingularity conditions on the curve $\hat{\mathcal{C}}$ Hitchin was able to prove all monopoles could be obtained by this approach Hit83. Bringing methods from integrable systems to bear upon the construction of solutions to Nahm's equations for the gauge group $S U(2)$ Ercolani and Sinha ES89] later showed how one could solve (a gauge transform of) the Nahm equations in terms of a Baker-Akhiezer function for the curve $\hat{\mathcal{C}}$.

Despite the many striking results now obtained, disappointingly few explicit solutions are known. This paper is part of a longer reappraisal of this work, seeking to understand where the difficulties in implementation lie and developing new techniques to address them. Throughout we shall focus on $S U(2)$ BPS monopoles and this paper will deal with curves $\hat{\mathcal{C}}$ of the form

$$
\eta^{3}+\chi\left(\zeta^{6}+b \zeta^{3}-1\right)=0
$$

where $\chi, b$ are certain real parameters. Such a curve is of genus 4 and could describe a charge three monopole. Two types of problem arise (that will be made more precise below). The first is that the curve (1.1) is subject to a set of constraints whereby the periods of a meromorphic differential on the curve are specified. This type of constraint arises in many other settings as well, for example when specifying the filling fractions of a curve in the AdS/CFT correspondence. Such constraints are transcendental in nature and the number of cases where they have be solved explicitly is rather limited. This is certainly an area that needs to be studied more. For the curve (1.1) a countable number of solutions to this constraint have been obtained. The second type of problem arises in implementing a constraint that may be expressed as the vanishing of a real one parameter family of cohomologies of certain line bundles, $H^{0}\left(\hat{\mathcal{C}}, L^{\lambda}(n-2)\right)=0$ for $\lambda \in(0,2)$. Viewing the line bundles as points on the Jacobian this is equivalent to a real line segment not intersecting the theta divisor $\Theta$ of the curve. Indeed there are sections for $\lambda=0,2$ and the flow is periodic $(\bmod 2)$ in $\lambda$ and so we are interested in the number of times a real line intersects $\Theta$. While techniques exist that count the number of intersections of a complex line with the theta divisor we are unaware of anything comparable in the real setting. We establish here that of the countable number of solutions to the second constraint that

Theorem 1. The only curves of the family (1.1) that yield BPS monopoles correspond to tetrahedrally symmetric monopoles. These have $b= \pm 5 \sqrt{2}, \chi^{\frac{1}{3}}=-\frac{1}{6} \frac{\Gamma\left(\frac{1}{6}\right) \Gamma\left(\frac{1}{3}\right)}{2^{\frac{1}{6}} \pi^{\frac{1}{2}}}$.

An outline of the paper is as follows. In section 2 we will recall the constraints on the curve $\hat{\mathcal{C}}$ that are equivalent for a monopole to exist. We shall then describe the curve (1.1) in more detail and make concrete these constraints for this curve. This will entail a description of the homology and period matrix for the curve. At this stage we will have reduced the problem to properties of the theta function for the genus 4 curve $\hat{\mathcal{C}}$. (Our theta function conventions are given in Appendix A.) Now the curve (1.1) (and indeed the more general curve $\eta^{3}+\alpha \eta \zeta^{2}+\chi\left(\zeta^{6}+b \zeta^{3}-1\right)=0$ that will be explored elsewhere) is invariant under 
the cyclic group $\mathrm{C}_{3}$ and we have a covering $\pi: \hat{\mathcal{C}} \rightarrow \mathcal{C}$ of a genus 2 curve $\mathcal{C}$. In section 3 we will describe a theorem of Fay and Accola that allows us to express the genus 4 theta functions in terms of genus 2 theta functions so reducing the problem to one of genus 2 theta functions. Then in section 4 we will use the reduction theory of Humbert to further reduce the problem to that of elliptic functions. At this stage we have reduced the initial problem of the existence of a BPS monopole to a question about the number of zeros of an elliptic function on an interval. Section 5 describes this in more detail. Although we have a stronger conjecture than we can prove, we are able to establish the theorem given above. We end with some final observations in section 6 .

\section{The Spectral Curve and its Constraints}

2.1. Hitchin Data. If $\zeta$ is the inhomogeneous coordinate on the Riemann sphere, and $(\zeta, \eta)$ are the standard local coordinates on $T \mathbb{P}^{1}$ (defined by $(\zeta, \eta) \rightarrow \eta \frac{d}{d \zeta}$ ), the spectral curve of a charge $n$ monopole $\hat{\mathcal{C}} \subset \mathrm{TP}^{1}$ may be expressed in the form

$$
P(\eta, \zeta)=\eta^{n}+\eta^{n-1} a_{1}(\zeta)+\ldots+\eta^{r} a_{n-r}(\zeta)+\ldots+\eta a_{n-1}(\zeta)+a_{n}(\zeta)=0
$$

where $a_{r}(\zeta)$ (for $1 \leq r \leq n$ ) is a polynomial in $\zeta$ of maximum degree $2 r$. We may view $\hat{\mathcal{C}}$ as an $n$-fold branched cover of $\mathbb{P}^{1}$ and (by a rotation if necessary) we may assume $n$ distinct preimages $\left\{\infty_{k}\right\}_{k=1}^{n}$ of the point $\zeta=\infty$. The form of the curve means that $\eta / \zeta \sim \rho_{k} \zeta$ at $\infty_{k}$. For a generic $n$-monopole the spectral curve is irreducible and has genus $g_{\hat{\mathcal{C}}}=(n-1)^{2}$. We will denote by $\left\{\hat{\mathfrak{a}}_{k}, \hat{\mathfrak{b}}_{k}\right\}_{k=1}^{g_{\hat{\mathcal{C}}}}$ a canonical homology basis of $\hat{\mathcal{C}}$.

The Hitchin data constrains the curve $\hat{\mathcal{C}}$ explicitly in terms of the polynomial $P(\eta, \zeta)$ and implicitly in terms of the behaviour of various line bundles on $\hat{\mathcal{C}}$. If the homogeneous coordinates of $\mathbb{P}^{1}$ are $\left[\zeta_{0}, \zeta_{1}\right]$ we consider the standard covering of this by the open sets $U_{0}=\left\{\left[\zeta_{0}, \zeta_{1}\right] \mid \zeta_{0} \neq 0\right\}$ and $U_{1}=\left\{\left[\zeta_{0}, \zeta_{1}\right] \mid \zeta_{1} \neq 0\right\}$, with $\zeta=\zeta_{1} / \zeta_{0}$ the usual coordinate on $U_{0}$. We will denote by $\hat{U}_{0,1}$ the pre-images of these sets under the projection map $\pi: T \mathbb{P}^{1} \rightarrow \mathbb{P}^{1}$. Let $L^{\lambda}$ denote the holomorphic line bundle on $T \mathbb{P}^{1}$ defined by the transition function $g_{01}=\exp (-\lambda \eta / \zeta)$ on $\hat{U}_{0} \cap \hat{U}_{1}$, and let $L^{\lambda}(m) \equiv L^{\lambda} \otimes \pi^{*} \mathcal{O}(m)$ be similarly defined in terms of the transition function $g_{01}=\zeta^{m} \exp (-\lambda \eta / \zeta)$. A holomorphic section of such line bundles is given in terms of holomorphic functions $f_{\alpha}$ on $\hat{U}_{\alpha}$ satisfying $f_{\alpha}=g_{\alpha \beta} f_{\beta}$. We denote line bundles on $\mathcal{C}$ in the same way, where now we have holomorphic functions $f_{\alpha}$ defined on $\mathcal{C} \cap \hat{U}_{\alpha}$.

The Hitchin data constrains the curve to satisfy:

H1: The curve $\hat{\mathcal{C}}$ is real with respect to the standard real structure on $T \mathbb{P}^{1}$ (the antiholomorphic involution defined by reversing the orientation of the lines in $\mathbb{R}^{3}$ ),

$$
\tau:(\zeta, \eta) \mapsto\left(-\frac{1}{\bar{\zeta}},-\frac{\bar{\eta}}{\bar{\zeta}^{2}}\right)
$$

H2: $L^{2}$ is trivial on $\hat{\mathcal{C}}$ and $L(n-1)$ is real.

H3: $H^{0}\left(\hat{\mathcal{C}}, L^{\lambda}(n-2)\right)=0$ for $\lambda \in(0,2)$.

Only the first of these constraints is immediate to implement. The reality of the curve means the coefficients of (2.1) satisfy

$$
a_{r}(\zeta)=(-1)^{r} \zeta^{2 r} \overline{a_{r}\left(-\frac{1}{\bar{\zeta}}\right)}
$$


For the curve (1.1) this is why $\chi$ and $b$ are real. Ercolani and Sinha show the reality of $L(n-1)$ within the Baker-Akhiezer function setting and BE06] implements this in terms of theta functions on the curve. The triviality of $L^{2}$ means that there exists a nowhere-vanishing holomorphic section. In terms of the open sets $\hat{U}_{0,1}$ we will have two, nowhere-vanishing holomorphic functions, $f_{0}$ on $\hat{U}_{0} \cap \hat{\mathcal{C}}$ and $f_{1}$ on $\hat{U}_{1} \cap \hat{\mathcal{C}}$, such that on the intersection

$$
f_{0}(\eta, \zeta)=\exp \left\{-2 \frac{\eta}{\zeta}\right\} f_{1}(\eta, \zeta)
$$

Consideration of the logarithmic derivative of (2.4) shows that, in order to avoid essential singularities, we must have

$$
\operatorname{dlog} f_{0}(P)=\left(\frac{2 \rho_{k}}{t^{2}}+O(1)\right) d t, \quad \text { at } \quad P \rightarrow \infty_{k},
$$

where $t=1 / \zeta$ is a local parameter. Ercolani and Sinha introduced the normalized meromorphic differential $\gamma_{\infty}$ whose pole behaviour is that of $\frac{1}{2} \operatorname{dlog} f_{0}(P)$ and whose $\mathfrak{a}$-periods vanish. In terms of the vector of $\mathfrak{b}$-periods we have

Lemma 2 (Ercolani-Sinha Constraints). The following are equivalent:

(1) $L^{2}$ is trivial on $\hat{\mathcal{C}}$.

(2) $2 \widehat{\boldsymbol{U}} \in \Lambda \Longleftrightarrow \widehat{\boldsymbol{U}}=\frac{1}{2 \pi \imath}\left(\oint_{\hat{\mathfrak{b}}_{1}} \gamma_{\infty}, \ldots, \oint_{\hat{\mathfrak{b}}_{g_{\mathcal{C}}}} \gamma_{\infty}\right)=\frac{1}{2} \boldsymbol{n}+\frac{1}{2} \hat{\tau} \boldsymbol{m}$ where $\boldsymbol{n}, \boldsymbol{m} \in \mathbb{Z}^{\hat{g}}$.

(3) There exists a 1-cycle $\mathfrak{c}=\boldsymbol{n} \cdot \hat{\mathfrak{a}}+\boldsymbol{m} \cdot \hat{\mathfrak{b}}$ such that $\oint_{\mathfrak{c}} \Omega=-2 \beta_{0}$ for every holomorphic differential $\Omega=\frac{\beta_{0} \eta^{n-2}+\beta_{1}(\zeta) \eta^{n-3}+\ldots+\beta_{n-2}(\zeta)}{\frac{\partial P}{\partial \eta}} d \zeta$, where $\beta_{j}(\zeta)$ is a polynomial of degree at most $2 j$ in $\zeta$.

Here $\Lambda$ is the period lattice of $\hat{\mathcal{C}}$ and $\hat{\tau}$ the $\mathfrak{a}$-normalized period matrix. Ercolani and Sinha established the equivalence of (1) and (2) while the dual form of the Ercolani-Sinha constraints (3) was given by Houghton, Manton and Ramão HMR00. If the anti-holomorphic involution $\tau$ induces an action $M_{\tau}$ on the homology, $\left(\begin{array}{l}\tau_{*} \hat{\mathfrak{a}} \\ \tau_{*} \hat{\mathfrak{b}}\end{array}\right)=M_{\tau}\left(\begin{array}{l}\hat{\mathfrak{a}} \\ \hat{\mathfrak{b}}\end{array}\right)$, then we have $M_{\tau} J M_{\tau}=-J$, where $J$ is the standard symplectic structure, and

Corollary 3. $\tau_{*} \mathfrak{c}=-\mathfrak{c}$ or $2 \widehat{\mathbf{U}} M_{\tau}=\left(\begin{array}{ll}\mathbf{n} & \mathbf{m}\end{array}\right) M_{\tau}=-\left(\begin{array}{ll}\mathbf{n} & \mathbf{m}\end{array}\right)$.

The Ercolani-Sinha constraints place $g_{\hat{\mathcal{C}}}$ transcendental constraints on the spectral curve $\hat{\mathcal{C}}$ and, as noted in the introduction, solving these is a major difficulty in implementing this theory.

We have yet to discuss H3, the implementation of which is the second type of problem mentioned in the introduction. Here $L^{\lambda}(n-2)$ is a degree $g_{\hat{\mathcal{C}}}-1$ line bundle so using Riemann's vanishing theorem for line bundles $\mathcal{L}$ of this degree, multiplicity $\mathcal{L}_{\mathcal{L}} \theta=$ $\operatorname{dim} H^{0}(\mathcal{C}, \mathcal{O}(\mathcal{L}))$, we see that $L^{\lambda}(n-2)$ does not lie in the theta divisor for $\lambda \in(0,2)$. In [BE06] we establish that

Lemma 4. Let $\widetilde{\boldsymbol{K}}=\boldsymbol{K}+\boldsymbol{\phi}\left((n-2) \sum_{k=1}^{n} \infty_{k}\right)$ where $\boldsymbol{K}$ is the vector of Riemann constants and $\phi$ the Abel-Jacobi map. Then

$$
H^{0}\left(\hat{\mathcal{C}}, L^{\lambda}(n-2)\right) \neq 0 \Longleftrightarrow \theta(\lambda \widehat{\boldsymbol{U}}-\widetilde{\boldsymbol{K}} \mid \hat{\tau})=0
$$

for $\lambda \in(0,2)$, where $\theta$ is Riemann's theta function for the curve $\hat{\mathcal{C}}$. 
Thus the second problem is to determine when the (real) line $\lambda \widehat{\boldsymbol{U}}-\widetilde{\boldsymbol{K}}$ intersects the theta divisor $\Theta$. We note the following properties of the vector $\widetilde{\boldsymbol{K}}$,

\section{Lemma 5 .}

- $\widetilde{\boldsymbol{K}}$ is independent of the choice of base point of the Abel map.

- $2 \widetilde{\boldsymbol{K}} \in \Lambda$.

- For $n \geq 3$ then $\widetilde{\boldsymbol{K}} \in \Theta_{\text {singular, }}$, the singular locus of the theta divisor.

- $\widehat{\boldsymbol{U}} \pm \widetilde{\boldsymbol{K}}$ is a non-singular even theta characteristic.

It is straightforward to see that $2 \widehat{\boldsymbol{U}} \neq 0$ and is a primitive vector in the period lattice. We also remark that because $\widetilde{\boldsymbol{K}}$ is a half-period then

$$
\theta(\lambda \widehat{\boldsymbol{U}}-\widetilde{\boldsymbol{K}} \mid \hat{\tau})=0 \Longleftrightarrow \theta(\lambda \widehat{\boldsymbol{U}}+\widetilde{\boldsymbol{K}} \mid \hat{\tau})=0 .
$$

2.2. The curve and its properties. We will write the curve (1.1) in the form

$$
w^{3}=z^{6}+b z^{3}-1=\left(z^{3}-\alpha^{3}\right)\left(z^{3}+\frac{1}{\alpha^{3}}\right)
$$

to avoid various factors, where $(z, w)=\left(\zeta,-\eta / \chi^{1 / 3}\right)$ and $1 / \alpha^{3}=\left(b+\sqrt{b^{2}+4}\right) / 2$. With $\rho=e^{2 \imath \pi / 3}$ this curve has symmetries:

$$
\mathrm{R}:(z, w) \rightarrow(z, \rho w), \quad \sigma:(z, w) \rightarrow(\rho z, \rho w), \quad \mathrm{c}:(z, w) \rightarrow\left(-1 / z,-w / z^{2}\right) .
$$

These yield the group $C_{3} \times S_{3}$, with $C_{3}=<\mathrm{R} \mid \mathrm{R}^{3}=1>$ and $S_{3}=<\sigma, \mathrm{c} \mid \sigma^{3}=1$, $\mathrm{c}^{2}=$ $1, \mathrm{c} \sigma \mathrm{c}=\sigma^{2}>$. When $b= \pm 5 \sqrt{2}$, the dihedral symmetry $S_{3}$ is enlarged to tetrahedral symmetry by

$$
\mathrm{t}:(z, w) \rightarrow\left( \pm \frac{\sqrt{2} \mp z}{1 \pm \sqrt{2} z},-\frac{3 w}{(1 \pm \sqrt{2} z)^{2}}\right), \quad \mathrm{t}^{2}=1,
$$

with $A_{4}$ being generated by $\sigma$ and t.

The paper BE06 chose an homology basis $\left\{\hat{\mathfrak{a}}_{i}^{s}, \hat{\mathfrak{b}}_{i}^{s}\right\}$ reflecting the symmetry R: $\mathrm{R}\left(\hat{\mathfrak{b}}_{i}^{s}\right)=$ $-\hat{\mathfrak{a}}_{i}^{s}(i=1,2,3)$ and $\mathrm{R}\left(\hat{\mathfrak{b}}_{4}^{s}\right)=\hat{\mathfrak{a}}_{4}^{s}$. If we order the branch points $\left\{\lambda_{1}, \ldots, \lambda_{6}\right\}$ in terms of increasing argument and denote by $\gamma_{k}\left(z_{i}, z_{j}\right)$ the oriented path going from $P_{i}=\left(z_{i}, w_{i}\right)$ to $P_{j}=\left(z_{j}, w_{j}\right)$ in the $k$-th sheet we may express these cycles as

$$
\begin{array}{ll}
\hat{\mathfrak{a}}_{1}^{s}=\gamma_{1}\left(\lambda_{2}, \lambda_{1}\right)+\gamma_{2}\left(\lambda_{1}, \lambda_{2}\right), & \hat{\mathfrak{b}}_{1}^{s}=\gamma_{1}\left(\lambda_{2}, \lambda_{1}\right)+\gamma_{3}\left(\lambda_{1}, \lambda_{2}\right), \\
\hat{\mathfrak{a}}_{2}^{s}=\gamma_{1}\left(\lambda_{4}, \lambda_{3}\right)+\gamma_{2}\left(\lambda_{3}, \lambda_{4}\right), & \hat{\mathfrak{b}}_{2}^{s}=\gamma_{1}\left(\lambda_{4}, \lambda_{3}\right)+\gamma_{3}\left(\lambda_{3}, \lambda_{4}\right), \\
\hat{\mathfrak{a}}_{3}^{s}=\gamma_{1}\left(\lambda_{6}, \lambda_{5}\right)+\gamma_{2}\left(\lambda_{5}, \lambda_{6}\right), & \hat{\mathfrak{b}}_{3}^{s}=\gamma_{1}\left(\lambda_{6}, \lambda_{5}\right)+\gamma_{3}\left(\lambda_{5}, \lambda_{6}\right), \\
\hat{\mathfrak{a}}_{4}^{s}=\gamma_{3}\left(\lambda_{2}, \lambda_{1}\right)+\gamma_{2}\left(\lambda_{1}, \lambda_{5}\right)+\gamma_{3}\left(\lambda_{5}, \lambda_{6}\right)+\gamma_{1}\left(\lambda_{6}, \lambda_{2}\right), \\
\hat{\mathfrak{b}}_{4}^{s}=\gamma_{2}\left(\lambda_{2}, \lambda_{1}\right)+\gamma_{3}\left(\lambda_{6}, \lambda_{2}\right)+\gamma_{2}\left(\lambda_{5}, \lambda_{6}\right)+\gamma_{1}\left(\lambda_{1}, \lambda_{5}\right) .
\end{array}
$$

This is shown in Figure 1(a). In the next section we shall choose a homology basis reflecting the symmetry $\sigma$ in order to use the results of Fay and Accola. Let us fix the following lexicographical ordering of independent canonical holomorphic differentials of $\hat{\mathcal{C}}$,

$$
d u_{1}=\frac{d z}{w}, \quad d u_{2}=\frac{d z}{w^{2}}, \quad d u_{3}=\frac{z d z}{w^{2}}, \quad d u_{4}=\frac{z^{2} d z}{w^{2}} .
$$

\footnotetext{
${ }^{1}$ The conventions of the paper [BE06] were such that the $\mathfrak{b}$-normalized period matrix was positive definite. We must change the relative orientation of the $\mathfrak{a}$-cycles and $\mathfrak{b}$-cycles to obtain the positive definite $\mathfrak{a}$ normalized period matrix used in this paper.
} 
Then the symmetry $\mathrm{R}$ together with the Riemann bilinear relations shows that the period matrix for $\hat{\mathcal{C}}$ may be expressed in terms of just the four periods

$$
\boldsymbol{x}=\left(x_{1}, x_{2}, x_{3}, x_{4}\right)^{T}=\left(\oint_{\mathfrak{a}_{1}} d u_{1}, \ldots, \oint_{\mathfrak{a}_{4}} d u_{1}\right)^{T} .
$$

Following Wellstein Wel899] and Matsumoto Mat01 we find

Proposition 6. Let $\hat{\mathcal{C}}$ be the triple covering of $\mathbb{P}^{1}$ with six distinct point $\lambda_{1}, \ldots, \lambda_{6}$,

$$
w^{3}=\prod_{i=1}^{6}\left(z-\lambda_{i}\right) .
$$

Then the a-normalized Riemann period matrix is of the form

$$
\hat{\tau}^{s}=\rho^{2}\left(H+\left(\rho^{2}-1\right) \frac{\boldsymbol{x} \boldsymbol{x}^{T}}{\boldsymbol{x}^{T} H \boldsymbol{x}}\right),
$$

where $H=\operatorname{diag}(1,1,1,-1)$. Then $\hat{\tau}^{s}$ is positive definite if and only if

$$
\overline{\boldsymbol{x}}^{T} H \boldsymbol{x}<0 .
$$

In fact the symmetry of (2.8) means that $x_{2}=\rho x_{1}, x_{3}=\rho^{2} x_{1}$, and only two periods need be computed. Choosing the first sheet so that $w=\sqrt[3]{\left(z^{3}-\alpha^{3}\right)\left(z^{3}+1 / \alpha^{3}\right)}$ is negative and real on the real $z$-axis between the branch points $(-1 / \alpha, \alpha)$ these may be expressed in terms of the integrals computed on the first sheet

$$
\begin{aligned}
& \mathcal{I}_{1}(\alpha)=\int_{0}^{\alpha} \frac{d z}{w}=-\frac{2 \pi \sqrt{3} \alpha}{9}{ }_{2} F_{1}\left(\frac{1}{3}, \frac{1}{3} ; 1 ;-\alpha^{6}\right), \\
& \mathcal{J}_{1}(\alpha)=\int_{0}^{-1 / \alpha} \frac{d z}{w}=\frac{2 \pi \sqrt{3}}{9 \alpha}{ }_{2} F_{1}\left(\frac{1}{3}, \frac{1}{3} ; 1 ;-\alpha^{-6}\right) .
\end{aligned}
$$

Here ${ }_{2} F_{1}(a, b ; c ; z)$ is the standard Gauss hypergeometric function and we may express the periods as $x_{1}=-\left(2 \mathcal{J}_{1}+\mathcal{I}_{1}\right) \rho-2 \mathcal{I}_{1}-\mathcal{J}_{1}$ and $x_{4}=3\left(\mathcal{J}_{1}-\mathcal{I}_{1}\right) \rho+3 \mathcal{J}_{1}$. Thus the period matrix may be expressed via (2.12) as rational expressions in terms of $x_{1}$ and $x_{4}$, or equivalently in terms of $\mathcal{I}_{1}$ and $\mathcal{J}_{1}$.

Now the Ercolani-Sinha constraints place constraints on the periods. These were solved in BE06, to give

Proposition 7. To each pair of relatively prime integers $(m, n)=1$ for which

$$
(m+n)(m-2 n)<0
$$


Ercolani-Sinha constraints for the curve (2.8) as follows. First we solve for t, where

$$
\frac{2 n-m}{m+n}=\frac{{ }_{2} F_{1}\left(\frac{1}{3}, \frac{2}{3} ; 1, t\right)}{{ }_{2} F_{1}\left(\frac{1}{3}, \frac{2}{3} ; 1,1-t\right)} \text {. }
$$

Then

$$
b=\frac{1-2 t}{\sqrt{t(1-t)}}, \quad t=\frac{-b+\sqrt{b^{2}+4}}{2 \sqrt{b^{2}+4}}
$$

\footnotetext{
${ }^{2}$ Note that $\boldsymbol{m}$ differs by a sign from that of [BE06] because we are working with $\mathfrak{a}$-normalized quantities and their attendant orientations (see the previous footnote).
} 
and we obtain $\chi$ from

$$
\chi^{\frac{1}{3}}=-(n+m) \frac{2 \pi}{3 \sqrt{3}} \frac{\alpha}{\left(1+\alpha^{6}\right)^{\frac{1}{3}}}{ }_{2} F_{1}\left(\frac{1}{3}, \frac{2}{3} ; 1, t\right)
$$

with $\alpha^{6}=t /(1-t)$.

Remarkably one may solve the transcendental equation (2.16) using a theory developed to explain various formulae of Ramanujan BBG95, Ber98. We shall not need any examples beyond those of [BE06]. At this stage one finds that the period matrix for a curve (2.8) satisfying $\mathbf{H 1}$ and $\mathbf{H 2}$ may be expressed in terms in terms of the quantity

$$
\mathcal{R}=\frac{\mathcal{I}_{1}(\alpha)}{\mathcal{J}_{1}(\alpha)}=\frac{m-2 n}{m+n} .
$$

We note the following symmetries that preserve the constraints on $(m, n)$,

$$
(m, n) \mapsto(-m,-n), \quad \mathcal{R} \mapsto \mathcal{R} ; \quad(m, n) \mapsto(n-m, n), \quad \mathcal{R} \mapsto \frac{1}{\mathcal{R}} .
$$

The remaining problem is to determine those allowed $(n, m)$ which also satisfy $\mathbf{H 3}$. To make use of our alternative characterisation (2.6) we record

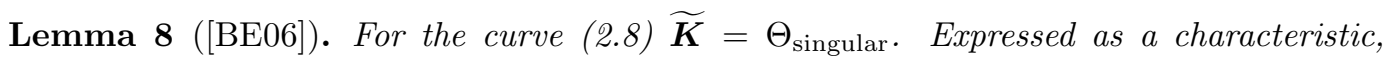
$\widetilde{\boldsymbol{K}}=\frac{1}{2}\left[\begin{array}{llll}1 & 1 & 1 & 1 \\ 1 & 1 & 1 & 1\end{array}\right]$.

Remark: Because in the case under consideration $\widetilde{\boldsymbol{K}}$ is an even half-period the function the function $\theta(\lambda \widehat{\boldsymbol{U}} \pm \widetilde{\boldsymbol{K}}, \hat{\tau})$ vanishes to second order at least at the points $\lambda=0$ and $\lambda=2$

$$
\begin{aligned}
& \left.\theta(\lambda \widehat{\boldsymbol{U}} \pm \widetilde{\boldsymbol{K}}, \hat{\tau})\right|_{\lambda \sim 0}=\partial_{\widehat{\boldsymbol{U}}}^{2} \theta(\widehat{\boldsymbol{K}} ; \widehat{\tau}) \lambda^{2}+O\left(\lambda^{4}\right), \\
& \left.\theta(\lambda \widehat{\boldsymbol{U}} \pm \widetilde{\boldsymbol{K}}, \hat{\tau})\right|_{\lambda \sim 2}=\partial_{\widehat{\boldsymbol{U}}}^{2} \theta(\widehat{\boldsymbol{K}} ; \widehat{\tau})(\lambda-2)^{2}+O\left((\lambda-2)^{4}\right) .
\end{aligned}
$$

We shall see that in fact it vanishes to order 4 .

Finally we note some of the coverings associated with the curve $\hat{\mathcal{C}}$.

Lemma 9. The action of $\sigma$ on the curve (2.8) yields the unramified covering $\pi: \widehat{\mathcal{C}} \rightarrow \mathcal{C}:=$ $\widehat{\mathcal{C}} / C_{3}$, where $\mathcal{C}$ is the genus 2 hyperelliptic curve

$$
\mathcal{C}=\left\{(\mu, \nu) \mid \nu^{2}=\left(\mu^{3}+b\right)^{2}+4\right\},
$$

with $\nu=z^{3}+1 / z^{3}$ and $\mu=-w / z$. Further, $\mathcal{C}$ two-sheetedly covers the two elliptic curves $\mathcal{E}_{ \pm}$

$$
\mathcal{E}_{ \pm}=\left\{\left(z_{ \pm}, w_{ \pm}\right) \mid w_{ \pm}^{2}=z_{ \pm}\left(1-z_{ \pm}\right)\left(1-k_{ \pm}^{2} z_{ \pm}\right)\right\}
$$

where

$$
\begin{aligned}
z_{ \pm} & =\frac{K^{2}-L^{2}}{K^{2}-\rho L^{2}} \frac{K-\mu}{\rho K-\mu} \frac{L^{2}-K \mu}{L^{2}-K \rho \mu} \\
w_{ \pm} & =-\imath \sqrt{2+\rho} \sqrt{\frac{L \pm K}{L \mp K}} \frac{K^{2}}{L} \frac{L^{2}-\rho K^{2}}{\rho L^{2}-K^{2}} \frac{\nu(L \mp \mu)}{(\mu-\rho K)^{2}\left(L^{2}-\rho K \mu\right)^{2}} .
\end{aligned}
$$

With $M=K / L, K=(2 \imath-b)^{\frac{1}{3}}$ and $L=\left(b^{2}+4\right)^{\frac{1}{6}}$ the Jacobi moduli $k_{ \pm}$are given by

$$
k_{ \pm}^{2}=-\frac{\rho(\rho M \pm 1)(\rho M \mp 1)^{3}}{(M \pm 1)(M \mp 1)^{3}} .
$$




\section{FAY-ACCOLA FACTORIZATION}

Thus far we have recalled earlier works: a countable putative family of spectral curves for $S U(2)$ BPS monopoles has been produced together with their period matrices and the vector $\widetilde{\boldsymbol{K}}$ but it remains to discuss the Hitchin constraint H3. The formulation of this constraint (2.6) is in terms of genus 4 theta functions and in this section we wish to reduce this to questions of genus 2 theta functions making use of a remarkable factorization theorem due to Accola and Fay Acc71, Fay73 and also observed by Mumford. Let $\pi: \hat{\mathcal{C}} \rightarrow \mathcal{C}$ be a cyclic unramified covering. The map $\pi$ leads to a map $\pi^{*}: \operatorname{Jac}(\mathcal{C}) \rightarrow \operatorname{Jac}(\hat{\mathcal{C}})$ which may be lifted to $\pi^{*}: \mathbb{C}^{g} \rightarrow \mathbb{C}^{\hat{g}}$. When $\hat{z}=\pi^{*} z$ the theta functions on $\hat{\mathcal{C}}$ and $\mathcal{C}$ are related by this factorization theorem. We shall now describe this theorem in the monopole setting.

Previously we have considered the symmetry $\mathrm{R}$ of the spectral curve. Now we shall focus on the cyclic symmetry $\sigma, \mathrm{C}_{3}=<\sigma \mid \sigma^{3}=1>$ and the unramified covering $\pi$ : $\widehat{\mathcal{C}} \rightarrow \mathcal{C}:=\widehat{\mathcal{C}} / \mathrm{C}_{3}$ described in Lemma 9. (The same considerations apply to the curve $\eta^{3}+\alpha \eta \chi^{2}+\chi\left(\zeta^{6}+b \zeta^{3}-1\right)=0$ and more generally cyclically symmetric monopoles.) To implement this theory we need a different choice of homology basis to that described earlier which reflects this symmetry. We wish an homology basis $\left\{\hat{\mathfrak{a}}_{0}^{c}, \ldots, \hat{\mathfrak{a}}_{3}^{c} ; \hat{\mathfrak{b}}_{0}^{c}, \ldots, \hat{\mathfrak{b}}_{3}^{c}\right\}$ on $\widehat{\mathcal{C}}$ and $\left\{\mathfrak{a}_{0}, \mathfrak{a}_{1}, \mathfrak{b}_{0}, \mathfrak{b}_{1}\right\}$ on $\mathcal{C}$ satisfying (for $i=1,2,3$ )

$$
\sigma^{k}\left(\hat{\mathfrak{a}}_{i}^{c}\right)=\hat{\mathfrak{a}}_{i+k}^{c}, \sigma^{k}\left(\hat{\mathfrak{b}}_{i}^{c}\right)=\hat{\mathfrak{b}}_{i+k}^{c}, \sigma^{k}\left(\hat{\mathfrak{a}}_{0}^{c}\right) \sim \hat{\mathfrak{a}}_{0}^{c}, \sigma^{k}\left(\hat{\mathfrak{b}}_{0}^{c}\right)=\hat{\mathfrak{b}}_{0}^{c}, \quad k=1,2,3
$$

(that is $\sigma^{k}\left(\hat{\mathfrak{a}}_{0}^{c}\right)$ is homologous to $\hat{\mathfrak{a}}_{0}^{c}$ ) and such that

$$
\pi\left(\hat{\mathfrak{a}}_{i}^{c}\right)=\mathfrak{a}_{1}, \pi\left(\hat{\mathfrak{b}}_{i}^{c}\right)=\mathfrak{b}_{1}, \pi\left(\hat{\mathfrak{a}}_{0}^{c}\right)=\mathfrak{a}_{0}, \pi\left(\hat{\mathfrak{b}}_{0}^{c}\right)=3 \mathfrak{b}_{0}
$$

We may construct such a basis as follows. We take $\hat{\mathfrak{a}}_{1}^{c}=\hat{\mathfrak{a}}_{1}^{s}, \hat{\mathfrak{b}}_{1}^{c}=\hat{\mathfrak{b}}_{1}^{s}=-\mathrm{R}^{2} \hat{\mathfrak{a}}_{1}^{s}$ and $\hat{\mathfrak{a}}_{0}^{c}=\hat{\mathfrak{a}}_{4}^{s}$ and extend these by $\hat{\mathfrak{a}}_{i+k}^{c}=\sigma_{*}^{k}\left(\hat{\mathfrak{a}}_{i}^{c}\right)$ and $\hat{\mathfrak{b}}_{i+k}^{c}=\sigma_{*}^{k}\left(\hat{\mathfrak{b}}_{i}^{c}\right)$. Thus $\hat{\mathfrak{a}}_{i+k}^{s}=\mathrm{R}^{2(i+k-1)} \sigma_{*}^{k}\left(\hat{\mathfrak{a}}_{i}^{c}\right)$, and $\hat{\mathfrak{b}}_{i+k}^{s}=\mathrm{R}^{2(i+k-1)} \sigma_{*}^{k}\left(\hat{\mathfrak{b}}_{i}^{c}\right)$. At this stage we have defined the cyclic cycles $\hat{\mathfrak{a}}_{i}^{c}, \hat{\mathfrak{b}}_{i}^{c}(i=1,2,3)$ together with $\hat{\mathfrak{a}}_{0}^{c}$. We complete the homology basis by seeking an invariant cycle $\hat{\mathfrak{b}}_{0}^{c}$ and define the cycles on $\mathcal{C}$ in terms of these. Such a basis is exhibited in Figure 1(b). If we take as ordered bases $\left\{\hat{\gamma}_{i}^{s}\right\}=\left\{\hat{\mathfrak{a}}_{1}^{s}, \ldots, \hat{\mathfrak{a}}_{4}^{s} ; \hat{\mathfrak{b}}_{1}^{s}, \ldots, \hat{\mathfrak{b}}_{4}^{s}\right\}$ and $\left\{\hat{\gamma}_{i}^{c}\right\}=\left\{\hat{\mathfrak{a}}_{1}^{c}, \ldots, \hat{\mathfrak{a}}_{3}^{c}, \hat{\mathfrak{a}}_{0}^{c} ; \hat{\mathfrak{b}}_{1}^{c}, \ldots, \hat{\mathfrak{b}}_{3}^{c}, \hat{\mathfrak{b}}_{0}^{c}\right\}$ then $\hat{\gamma}_{i}^{c}=\mathfrak{S} \hat{\gamma}_{i}^{s}$ where $\mathfrak{S}$ is the symplectic matrix

$$
\mathfrak{S}=\left(\begin{array}{cccccccc}
1 & 0 & 0 & 0 & 0 & 0 & 0 & 0 \\
0 & -1 & 0 & 0 & 0 & 1 & 0 & 0 \\
0 & 0 & 0 & 0 & 0 & 0 & -1 & 0 \\
0 & 0 & 0 & 1 & 0 & 0 & 0 & 0 \\
0 & 0 & 0 & 0 & 1 & 0 & 0 & 0 \\
0 & -1 & 0 & 0 & 0 & 0 & 0 & 0 \\
0 & 0 & 1 & 0 & 0 & 0 & -1 & 0 \\
0 & 0 & 0 & -1 & 0 & 0 & 0 & 1
\end{array}\right):=\left(\begin{array}{cc}
A & B \\
C & D
\end{array}\right) \in \operatorname{Sp}(8, \mathbb{Z})
$$

For example

$$
\hat{\mathfrak{a}}_{2}^{c}=\sigma \hat{\mathfrak{a}}_{1}^{c}=\sigma \hat{\mathfrak{a}}_{1}^{s}=\mathcal{R} \hat{\mathfrak{a}}_{2}^{s}=-\mathcal{R}^{2} \hat{\mathfrak{b}}_{2}^{s}=(1+\mathcal{R}) \hat{\mathfrak{b}}_{2}^{s}=-\hat{\mathfrak{a}}_{2}^{s}+\hat{\mathfrak{b}}_{2}^{s}
$$


Fay works with the ordered basis $\left\{\hat{\gamma}_{i}^{c}\right\}=\left\{\hat{\mathfrak{a}}_{0}^{c}, \hat{\mathfrak{a}}_{1}^{c}, \ldots, \hat{\mathfrak{a}}_{3}^{c} ; \hat{\mathfrak{b}}_{0}^{c}, \hat{\mathfrak{b}}_{1}^{c}, \ldots, \hat{\mathfrak{b}}_{3}^{c}\right\}$, this reordering being achieved (on both the $\mathfrak{a}$ and $\mathfrak{b}$-cycles) by

$$
\mathrm{S}:=\left(\begin{array}{llll}
0 & 0 & 0 & 1 \\
1 & 0 & 0 & 0 \\
0 & 1 & 0 & 0 \\
0 & 0 & 1 & 0
\end{array}\right) .
$$

We may again represent these cycles as integrals between branch points:

$$
\begin{aligned}
& \hat{\mathfrak{a}}_{1}^{c}=\gamma_{1}\left(\lambda_{2}, \lambda_{1}\right)+\gamma_{2}\left(\lambda_{1}, \lambda_{2}\right), \quad \hat{\mathfrak{b}}_{1}^{c}=\gamma_{1}\left(\lambda_{2}, \lambda_{1}\right)+\gamma_{3}\left(\lambda_{1}, \lambda_{2}\right), \\
& \hat{\mathfrak{a}}_{2}^{c}=\gamma_{2}\left(\lambda_{4}, \lambda_{3}\right)+\gamma_{3}\left(\lambda_{3}, \lambda_{4}\right), \quad \hat{\mathfrak{b}}_{2}^{c}=\gamma_{2}\left(\lambda_{4}, \lambda_{3}\right)+\gamma_{1}\left(\lambda_{3}, \lambda_{4}\right), \\
& \hat{\mathfrak{a}}_{3}^{c}=\gamma_{3}\left(\lambda_{6}, \lambda_{5}\right)+\gamma_{1}\left(\lambda_{5}, \lambda_{6}\right), \quad \hat{\mathfrak{b}}_{3}^{c}=\gamma_{3}\left(\lambda_{6}, \lambda_{5}\right)+\gamma_{2}\left(\lambda_{5}, \lambda_{6}\right), \\
& \hat{\mathfrak{a}}_{0}^{c}=\gamma_{3}\left(\lambda_{2}, \lambda_{1}\right)+\gamma_{2}\left(\lambda_{1}, \lambda_{5}\right)+\gamma_{3}\left(\lambda_{5}, \lambda_{6}\right)+\gamma_{1}\left(\lambda_{6}, \lambda_{2}\right), \\
& \hat{\mathfrak{b}}_{0}^{c}=\gamma_{3}\left(\lambda_{1}, \lambda_{2}\right)+\gamma_{1}\left(\lambda_{2}, \lambda_{5}\right)+\gamma_{2}\left(\lambda_{5}, \lambda_{6}\right)+\gamma_{3}\left(\lambda_{6}, \lambda_{3}\right)+\gamma_{1}\left(\lambda_{3}, \lambda_{4}\right)+\gamma_{2}\left(\lambda_{4}, \lambda_{1}\right) .
\end{aligned}
$$

With the cyclic homology basis just described we have

Theorem 10 (Fay-Accola). With respect to the ordered canonical homology bases $\left\{\hat{\gamma}_{i}^{c}\right\}=$ $\left\{\hat{\mathfrak{a}}_{0}^{c}, \hat{\mathfrak{a}}_{1}^{c}, \ldots, \hat{\mathfrak{a}}_{3}^{c} ; \hat{\mathfrak{b}}_{0}^{c}, \hat{\mathfrak{b}}_{1}^{c}, \ldots, \hat{\mathfrak{b}}_{3}^{c}\right\}$ and $\left\{\mathfrak{a}_{0}, \mathfrak{a}_{1}, \mathfrak{b}_{0}, \mathfrak{b}_{1}\right\}$ specified above then the $\mathfrak{a}$-normalized Riemann period matrices of $\hat{\mathcal{C}}$ and $\mathcal{C}$ take the respective forms

$$
\hat{\tau}^{c}=\left(\begin{array}{llll}
a & b & b & b \\
b & c & d & d \\
b & d & c & d \\
b & d & d & c
\end{array}\right), \quad \tau^{c}=\left(\begin{array}{cc}
\frac{1}{3} a & b \\
b & c+2 d
\end{array}\right) .
$$

Moreover for arbitrary $\boldsymbol{z}=\left(z_{0}, z_{1}\right) \in \mathbb{C}^{2}$ then $\pi^{*} \boldsymbol{z}=\hat{\boldsymbol{z}}=\left(3 z_{0}, z_{1}, z_{1}, z_{1}\right)$ and

$$
\frac{\theta\left(3 z_{0}, z_{1}, z_{1}, z_{1} ; \hat{\tau}^{c}\right)}{\prod_{k=0}^{2} \theta\left[\begin{array}{cc}
0 & 0 \\
\frac{k}{3} & 0
\end{array}\right]\left(z_{0}, z_{1} ; \tau^{c}\right)}=c_{0}\left(\widehat{\tau}^{c}\right)
$$

is a non-zero modular constant $c_{0}\left(\hat{\tau}^{c}\right)$ independent of $\boldsymbol{z}$.

In our setting we obtain

Proposition 11. The quantities $a, b, c, d$ appearing in the Fay-Accola theorem are expressible in terms of the holomorphic integrals $x_{1}, x_{4}$ (with $\rho^{3}=1$ ) as

$$
\begin{aligned}
a & =-\frac{6 x_{1}^{2}-x_{4}^{2}+\rho\left(3 x_{1}^{2}+x_{4}^{2}\right)}{3 x_{1}^{2}-x_{4}^{2}}, & b & =\frac{(1+2 \rho) x_{1} x_{4}}{3 x_{1}^{2}-x_{4}^{2}}, \\
c & =\frac{2 x_{1}^{2}-x_{4}^{2}+\rho\left(x_{1}^{2}-x_{4}^{2}\right)}{3 x_{1}^{2}-x_{4}^{2}}, & d & =-\frac{(1+2 \rho) x_{1}^{2}}{3 x_{1}^{2}-x_{4}^{2}} .
\end{aligned}
$$

Proof. If we perform the symplectic transformation of the period matrix (2.12) with the symplectic transformation (3.1), $\hat{\tau}^{s} \rightarrow\left(C+D \hat{\tau}^{s}\right)\left(A+B \hat{\tau}^{s}\right)^{-1}$, we obtain a period matrix of the form

$$
\left(\begin{array}{llll}
c & d & d & b \\
d & c & d & b \\
d & d & c & b \\
b & b & b & a
\end{array}\right)=\mathrm{S}^{-1} \hat{\tau}^{c} \mathrm{~S}
$$


the final result coming after conjugation by $\mathrm{S}$ to change the order of the homology basis to match that of Fay.

Again we see that the period matrix just depends on the ratio of $x_{1} / x_{4}$ or equivalently on $\mathcal{R}$. Now to make use of the Fay-Accola theorem we must show that the vectors $\widehat{\boldsymbol{U}}$ and $\widetilde{\boldsymbol{K}}$ may be obtained by pullback from $\operatorname{Jac}(\mathcal{C})$. To this end we have

Proposition 12. In the cyclic homology basis $\left\{\hat{\mathfrak{a}}_{0}^{c}, \ldots, \hat{\mathfrak{a}}_{3}^{c} ; \hat{\mathfrak{b}}_{0}^{c}, \ldots, \hat{\mathfrak{b}}_{3}^{c}\right\}$ the winding vector $\widehat{\boldsymbol{U}}$ and vector $\widetilde{\boldsymbol{K}}$ take the form

$$
\begin{aligned}
\widehat{\boldsymbol{U}} & =\left(\widehat{U}_{0}, \widehat{U}_{1}, \widehat{U}_{1}, \widehat{U}_{1}\right), \quad \widehat{U}_{0}=-\frac{C_{0} x_{4}}{3 x_{1}^{2}-x_{4}^{2}}, \quad \widehat{U}_{1}=\frac{C_{0} x_{1}}{3 x_{1}^{2}-x_{4}^{2}}, \\
\widetilde{\boldsymbol{K}} & =\left(\frac{1}{2}, \frac{1}{2}, \frac{1}{2}, \frac{1}{2}\right)+\left(\frac{1}{2}, \frac{1}{2}, \frac{1}{2}, \frac{1}{2}\right) \hat{\tau}^{c}=\left(\widetilde{K}_{0}, \widetilde{K}_{1}, \widetilde{K}_{1}, \widetilde{K}_{1}\right),
\end{aligned}
$$

where $C_{0}=-3(2 n-m)$. The winding vector is a half-period and the Ercolani-Sinha vector may be written $2 \widehat{\boldsymbol{U}}=\widehat{\boldsymbol{n}}+\widehat{\boldsymbol{m}} \hat{\tau}^{c}$ where

$$
(\widehat{\boldsymbol{n}}, \widehat{\boldsymbol{m}})=(5 n-m, n, n, n, 3 n,-m,-m,-m) .
$$

Proof. Using the explicit expression for the matrix $\mathcal{A}$ from [BE06] one has the vector $\widehat{\boldsymbol{U}}^{s}=$ $\nu(1,0,0,0) \mathcal{A}^{-1}$ and

$$
\widehat{\boldsymbol{U}}^{s}=-C_{0}\left(\frac{x_{1}}{x_{4}^{2}}, \frac{\rho x_{1}}{x_{4}^{2}}, \frac{\rho^{2} x_{1}}{x_{4}^{2}},-\frac{1}{x_{4}}\right) .
$$

Then with the symplectic transformation (3.1) $\widehat{\boldsymbol{U}}=\widehat{\boldsymbol{U}}^{s}\left(A+B \hat{\tau}^{s}\right)^{-1}$. The value of constant $C_{0}$ is found from the condition H2. Performing the symplectic transformation on the vector $(\boldsymbol{n}, \boldsymbol{m})$ given in Proposition 7 yields

$$
(\widehat{\boldsymbol{n}} \mathrm{S}, \widehat{\boldsymbol{m}} \mathrm{S})=(\boldsymbol{n}, \boldsymbol{m}) \mathfrak{S}^{-1}=(n, n, n, 5 n-m,-m,-m,-m, 3 n)
$$

and the result follows.

The only point to note is in the transformation of the vector of Riemann constants. This has two parts: the vector $\boldsymbol{K}$ transforms as a vector, $\boldsymbol{K} \rightarrow \boldsymbol{K}\left(A+B \hat{\tau}^{s}\right)^{-1}$; but in transforming the argument of a theta function function by a symplectic transformation the characteristics of the theta function also transform (see Appendix A), and a theta function with no characteristics may acquire characteristics. When dealing with Riemann's theta function (with vanishing characteristic) the acquired characteristics are typically placed in the transformed vector of Riemann constants. We do this here and find

$$
\widetilde{\boldsymbol{K}}=\left(\frac{1}{2} \ldots \frac{1}{2}\right) \mathfrak{S}^{-1}\left(\begin{array}{c}
1 \\
\widehat{\tau}^{c}
\end{array}\right)+\frac{1}{2}\left(\left(C D^{T}\right)_{0},\left(A B^{T}\right)_{0}\right)\left(\begin{array}{c}
1 \\
\widehat{\tau}^{c}
\end{array}\right)
$$

yielding the stated result.

The form of the vectors $\widehat{\boldsymbol{U}}$ and $\widetilde{\boldsymbol{K}}$ given in the theorem establishes

Corollary 13. $\widehat{\boldsymbol{U}}=\pi^{*}\left(\boldsymbol{U}^{*}\right)$ and $\widetilde{\boldsymbol{K}}=\pi^{*}\left(\boldsymbol{K}^{*}\right)$ where

$$
\boldsymbol{U}^{*}=\left(\frac{1}{3} \widehat{U}_{0}, \widehat{U}_{1}\right), \boldsymbol{K}^{*}=\left(\frac{1}{3} \widehat{K}_{0}, \widehat{K}_{1}\right) .
$$

Therefore we may employ the Fay-Accola result. Introduce the three functions

$$
f_{k}(\lambda)=\theta\left(\lambda \boldsymbol{U}^{*}+\boldsymbol{K}^{*}+k \boldsymbol{l}^{*} \mid \tau^{c}\right), \quad k=0,+1,-1, \quad \boldsymbol{l}^{*}=\left(\frac{1}{3}, 0\right) .
$$


Up to exponential factors these correspond to the three genus 2 theta functions with characteristics in the denominator of (3.4). Making use of (2.6, 2.7) we arrive at

Theorem 14. If $\lambda \in[0,2]$ then

$$
H^{0}\left(\hat{\mathcal{C}}, L^{\lambda}(n-2)\right) \neq 0 \Longleftrightarrow \theta(\lambda \widehat{\boldsymbol{U}} \pm \widetilde{\boldsymbol{K}} \mid \hat{\tau})=0 \Longleftrightarrow f_{k}(\lambda)=0
$$

for at least one $k \in\{0, \pm 1\}$.

At this stage we have reduced the question $\mathbf{H 3}$ to questions about various genus 2 theta functions and we shall look at these in the next section.

Remark: We note that the symplectic transformation

brings $\widehat{\tau}^{c}$ to the form

$$
\left(\begin{array}{cccccccc}
0 & 0 & 0 & 0 & 0 & 1 & 1 & 1 \\
0 & 0 & 0 & 0 & 1 & 0 & 0 & 0 \\
0 & 2 & -1 & -1 & 0 & 0 & 0 & 0 \\
0 & 0 & 0 & 0 & 0 & 0 & 1 & -1 \\
0 & -1 & 0 & 0 & 0 & 0 & 0 & 0 \\
-1 & 0 & 0 & 0 & 0 & 0 & 0 & 0 \\
0 & 0 & 0 & 0 & 0 & 0 & 0 & -1 \\
0 & 1 & -1 & 0 & 0 & 0 & 0 & 0
\end{array}\right)
$$

$$
\left(\begin{array}{cccc}
-\frac{1}{3} \frac{a}{-3 b^{2}+a c+2 a d} & \frac{b}{-3 b^{2}+a c+2 a d} & -\frac{1}{3} & 0 \\
\frac{b}{-3 b^{2}+a c+2 a d} & -\frac{c+2 d}{-3 b^{2}+a c+2 a d} & 0 & 0 \\
-\frac{1}{3} & 0 & \frac{c-d}{6} & \frac{1}{2} \\
0 & 0 & \frac{1}{2} & -\frac{1}{2} \frac{1}{c-d}
\end{array}\right)
$$

One may identify the top $2 \times 2$ block as conjugate to $-\tau^{c-1} / 3$. Using this one can use Weierstrass reduction to rewrite the genus 4 theta functions as in BE06.

\section{The Humbert VARIETy}

At this stage by using the Fay-Accola theorem we have reduced the question of the vanishing of the genus $4 \theta$-function $\theta\left(\lambda \widehat{\boldsymbol{U}}+\widetilde{\boldsymbol{K}} ; \widehat{\tau}^{c}\right)$ to that of the vanishing of the genus 2 $\theta$-functions $f_{k}(\lambda), k \in\{0, \pm 1\}$. We can in fact do better. In [BE06] it was observed that $\mathcal{C}$ covered two elliptic curves and we shall now exploit this geometry making use of ideas of Humbert expounded in Krazer Kra03 that we now recall.

Definition 1. The period matrix $\tau$ of a genus two algebraic curve $\mathcal{C}$ belongs to the Humbert variety $\mathcal{H}_{\Delta}$ associated with the symplectic invariant $\Delta$ if there exist integer $q_{i} \in \mathbb{Z}$ satisfying

$$
q_{1}+q_{2} \tau_{11}+q_{3} \tau_{12}+q_{4} \tau_{22}+q_{5}\left(\tau_{12}^{2}-\tau_{11} \tau_{22}\right)=0
$$

and

$$
q_{3}^{2}-4\left(q_{1} q_{5}+q_{2} q_{4}\right)=\Delta
$$

The curve $\mathcal{C}$ covers elliptic curves $\mathcal{E}_{ \pm}$if and only if $\Delta$ is a perfect square, $\Delta=h^{2} \geq 1$, $h \in \mathbb{N}$. Then the integer $h$ is the degree of the cover. 
Theorem 15 (Bierman-Humbert). Let $\tau \in \mathcal{H}_{\Delta}$ and $\Delta=h^{2}$. Then there exists a symplectic transformation $\mathfrak{S} \in \operatorname{Sp}(4, \mathbb{Z})$, such that

$$
\mathfrak{S} \circ \tau=\widetilde{\tau}=\left(\begin{array}{cc}
\widetilde{\tau}_{11} & \frac{1}{h} \\
\frac{1}{h} & \widetilde{\tau}_{22}
\end{array}\right)
$$

The transformation $\mathfrak{S}$ is given constructively and may be realized in a finite number of steps.

A modern proof of the theorem is given Mur94 revising that of Krazer Kra03.

When a $2 \times 2$ period matrix $\widetilde{\tau}$ has the structure (4.3) we may decompose the associated $\theta$-function as

$$
\theta\left(z_{1}, z_{2} \mid \widetilde{\tau}\right)=\sum_{k=0}^{h-1} \vartheta_{3}\left(z_{1}+\frac{k}{h} \mid \widetilde{\tau}_{1,1}\right) \theta\left[\begin{array}{c}
\frac{k}{h} \\
0
\end{array}\right]\left(h z_{2} \mid h^{2} \widetilde{\tau}_{2,2}\right), \quad h^{2}=\Delta .
$$

Here and below $\vartheta_{k}(z \mid \tau), k=1,2,3,4$ denote the Jacobi theta functions BE55.

Our case is relevant to the Humbert variety $\mathcal{H}_{4}$ that has received most study. The following is true.

Proposition 16. Let $\tau^{c}$ be the period matrix of the curve $\mathcal{C}$. Then $\tau^{c} \in \mathcal{H}_{4}$ with

$$
\widetilde{\tau}_{11}=\frac{1}{2} \frac{(\rho-1)\left(-3 x_{1}+2 x_{4}+\rho x_{4}\right)}{3 x_{1}-x_{4}+\rho x_{4}}, \quad \widetilde{\tau}_{22}=\frac{1}{6} \frac{(2+\rho)\left(-3 x_{1}-x_{4}+\rho x_{4}\right)}{3 x_{1}+2 x_{4}+\rho x_{4}} .
$$

Proof. Substituting the expressions we have for $a, b, c$ and $d$ in terms of $x_{1}$ and $x_{4}$ in the matrix equality

$$
\tau^{c}=\left(\begin{array}{cc}
\tau_{11} & \tau_{12} \\
\tau_{12} & \tau_{22}
\end{array}\right)=\left(\begin{array}{cc}
\frac{1}{3} a & b \\
b & c+2 d
\end{array}\right)
$$

we may eliminate $x_{1}$ and $x_{4}$ to obtain the two relations:

$$
\begin{aligned}
& 0=-2-\tau_{22}+3 \tau_{11}, \\
& 0=1-3 \tau_{11}-\tau_{12}^{2}+3 \tau_{11}^{2} .
\end{aligned}
$$

Using the first of these we may write $3 \tau_{11}^{2}=\tau_{11}\left(\tau_{22}+2\right)$ which leads to the second taking the form

$$
1-\tau_{11}+\tau_{11} \tau_{22}-\tau_{12}^{2}=0
$$

This is (4.1) with $q_{1}=1, q_{2}=-1, q_{3}=0, q_{4}=0, q_{5}=-1$ and the value of the invariant $\Delta=4$. (We remark that other possibilities may arise in the elimination process but we present only one resulting in $\Delta=4$.) Standard procedure [Kra03, BBE94 yields the symplectic transformation

$$
\mathfrak{S}=\left(\begin{array}{llll}
0 & 1 & 1 & 0 \\
1 & 1 & 0 & 1 \\
0 & 1 & 0 & 1 \\
0 & 0 & 1 & 0
\end{array}\right)=\left(\begin{array}{ll}
\alpha & \beta \\
\gamma & \delta
\end{array}\right) \in \operatorname{Sp}(4, \mathbb{Z})
$$

which reduces $\tau^{c}$ to the form (4.3) with $h=2$ and the stated identifications (4.5).

This proposition enables us to write the genus two theta functions and so the functions $f_{k}$ in terms of Jacobi $\theta$-functions,

$$
\theta\left(z_{1}, z_{2} \mid \widetilde{\tau}\right)=\vartheta_{3}\left(z_{1} \mid \widetilde{\tau}_{1,1}\right) \vartheta_{3}\left(2 z_{2} \mid 4 \widetilde{\tau}_{2,2}\right)+\vartheta_{3}\left(z_{1}+1 / 2 \mid \widetilde{\tau}_{1,1}\right) \vartheta_{2}\left(2 z_{2} \mid 4 \widetilde{\tau}_{2,2}\right) .
$$

We will need to transform the argument $\boldsymbol{z}=\lambda \boldsymbol{U}^{*}+\boldsymbol{K}^{*}+k \boldsymbol{l}^{*}$ using the transformation (4.8) but before doing so it is helpful to consider the moduli $\widetilde{\tau}_{11}$ and $\widetilde{\tau}_{22}$ and an additional link between them. As explained earlier, the periods $x_{1,4}$ are expressible in terms of the 
integrals $\mathcal{I}_{1}(\alpha)$ and $\mathcal{J}_{1}(\alpha)$ whose ratio (2.19) is constrained to be $\mathcal{R}$. Only the ratios of $x_{1,4}$ appear in (4.5) and we may replace these by $\mathcal{R}$,

$$
\widetilde{\tau}_{11}=\frac{1}{2} \frac{2+4 \rho+3 \mathcal{R}}{\mathcal{R}+1+2 \rho}=\frac{2 i \sqrt{3}+3 \mathcal{R}}{2(\mathcal{R}+i \sqrt{3})}, \quad \widetilde{\tau}_{22}=-\frac{1+2 \rho+3 \mathcal{R}}{6 \mathcal{R}}=-\frac{i \sqrt{3}+3 \mathcal{R}}{6 \mathcal{R}} .
$$

It is convenient to introduce the purely imaginary quantity (with positive imaginary part)

$$
\mathcal{T}=-\frac{2 \imath \sqrt{3}}{\mathcal{R}}=2 \imath \sqrt{3} \frac{n+m}{2 n-m} .
$$

In terms of this we have

$$
\widetilde{\tau}_{11}=1-\frac{1}{\mathcal{T}-2}, \quad \widetilde{\tau}_{22}=\frac{\mathcal{T}}{12}-\frac{1}{2}
$$

and the transformed arguments take the form

$$
\begin{aligned}
\boldsymbol{U}^{\prime} & =\boldsymbol{U}^{*}(\alpha+\beta \tau)^{-1}=\left(\frac{(-1+i \sqrt{3}) C_{0} \mathcal{T}}{36(\mathcal{T}-2)},-\frac{(3+i \sqrt{3}) C_{0} \mathcal{T}}{216}\right), \\
\boldsymbol{l}^{\prime} & =\boldsymbol{l}^{*}(\alpha+\beta \tau)^{-1}=\left(-\frac{(\mathcal{T}-3)}{3(\mathcal{T}-2)}, \frac{1}{6}\right), \\
\boldsymbol{K}^{\prime} & =\boldsymbol{K}^{*}(\alpha+\beta \tau)^{-1}+\frac{1}{2}\left(\left(\gamma \delta^{T}\right)_{0},\left(\alpha \beta^{T}\right)_{0}\right)\left(\begin{array}{c}
1 \\
\tau_{\mathfrak{a}}
\end{array}\right)=\left(\frac{4}{3}-\frac{1}{3(\mathcal{T}-2)}, \frac{\mathcal{T}}{12}-\frac{1}{6}\right) .
\end{aligned}
$$

Using (4.11, 4.9) and various substitutions the following proposition is established in Appendix B.

Proposition 17. For each pair of relatively prime integers $(m, n)=1$ for which $(2 n-$ $m)(n+m)>0$ let $\widehat{\boldsymbol{U}}$ be the Ercolani-Sinha vector and $\widehat{\tau}$ the period matrix of the genus 4 curve described above. Then the function $\theta(\lambda \widehat{\boldsymbol{U}}+\widehat{\boldsymbol{K}} \mid \widehat{\tau})$ vanishes for $\lambda \in[0,2]$ if and only if at least one of the three functions (with $k \in \mathbb{Z}$ )

$$
h_{k}(y):=\frac{\vartheta_{3}}{\vartheta_{2}}\left(i \sqrt{3} y+\frac{k \mathcal{T}}{3} \mid \mathcal{T}\right)+(-1)^{k} \frac{\vartheta_{2}}{\vartheta_{3}}\left(y+\frac{k}{3} \mid \frac{\mathcal{T}}{3}\right), \quad k=-1,0,1 \quad \bmod 3,
$$

also vanishes. Here $y:=y(\lambda)=\lambda(n+m) \rho / 3, \mathcal{T}=2 i \sqrt{3}(n+m) /(2 n-m)$ and $\frac{\vartheta_{3}}{\vartheta_{2}}(z \mid \mathcal{T})$ is shorthand for $\frac{\vartheta_{3}(z \mid \mathcal{T})}{\vartheta_{3}(z \mid \mathcal{T})}$. Further the functions $h_{k}$ satisfy

$$
\begin{aligned}
h_{k+3}(y) & =h_{k}(y), \quad h_{k}\left(y+\frac{2(n+m)}{3}\right)=h_{k-[n+m]}(y), \\
h_{k}\left(y+\frac{2(n+m)}{3} \rho\right) & = \begin{cases}(-1)^{n+m} h_{k-[n+m]}(y) & \text { if } m \text { even, } \\
(-1)^{k} h_{k-[n+m]}(y+\mathcal{T} / 2) & \text { if } m \text { odd },\end{cases} \\
h_{k}(y(\lambda+2)) & =0 \Longleftrightarrow h_{k-[n+m]}(y(\lambda))=0 .
\end{aligned}
$$

Therefore $h_{k}$ are elliptic functions with periods $2(n+m)$ and $4(n+m) \rho$. We also note that the zero divisors of $h_{k}(y)$ and $h_{k}(y+\mathcal{T} / 2)$ are the same.

Thus we have reduced the question of $\mathbf{H 3}$ to that of the zeros of the elliptic functions $h_{k}$. This theta function question is much simpler than the corresponding (much greater degree) theta function expressions of [BE06] which arose making use of Weierstrass-Poincaré reduction. Exploiting the geometry has greatly simplified the problem. We shall turn to the theta function question in the next section. 
Remark: We have an action of $\Gamma(2) \times \Gamma(2)$ on period matrices of the form $\left(\begin{array}{cc}\lambda_{1} & 1 / 2 \\ 1 / 2 & \lambda_{2}\end{array}\right)$ which may be associated to any genus 2 curve with extra involution distinct from the hyperelliptic involution. Here $\Gamma(2)=\left\{\left(\begin{array}{ll}a & b \\ c & d\end{array}\right) \in P S L(2, \mathbb{Z}) \mid a \equiv d \equiv 1(\bmod 2), b \equiv c \equiv 0(\bmod 2)\right\}$ has generators $\tau \mapsto \tau+2$ and $\tau \mapsto \frac{\tau}{1-2 \tau}$ and we have the exact sequence

$$
1 \rightarrow \Gamma(2) \rightarrow P S L(2, \mathbb{Z}) \rightarrow S_{3} \rightarrow 1 .
$$

To see this we observe that with the action $\left(\begin{array}{ll}A & B \\ C & D\end{array}\right): \tau \mapsto(C+D \tau)(A+B \tau)^{-1}$ we have

$$
\begin{aligned}
& s \quad\left(\begin{array}{cccc}
1 & 2 & -4 & 0 \\
0 & 1 & 0 & 0 \\
0 & 0 & 1 & 0 \\
0 & 1 & -2 & 1
\end{array}\right) \quad\left(\begin{array}{cc}
\lambda_{1} & 1 / 2 \\
1 / 2 & \lambda_{2}
\end{array}\right) \mapsto\left(\begin{array}{cc}
\frac{\lambda_{1}}{1-4 \lambda_{1}} & 1 / 2 \\
1 / 2 & \lambda_{2}
\end{array}\right) \\
& t \quad\left(\begin{array}{cccc}
1 & 0 & 0 & 0 \\
0 & 1 & 0 & 0 \\
1 & 0 & 1 & 0 \\
0 & 0 & 0 & 1
\end{array}\right) \quad\left(\begin{array}{cc}
\lambda_{1} & 1 / 2 \\
1 / 2 & \lambda_{2}
\end{array}\right) \mapsto\left(\begin{array}{cc}
\lambda_{1}+1 & 1 / 2 \\
1 / 2 & \lambda_{2}
\end{array}\right) \\
& \left(\begin{array}{cccc}
0 & 1 & 0 & 0 \\
1 & 0 & 0 & 0 \\
0 & 0 & 0 & 1 \\
0 & 0 & 1 & 0
\end{array}\right) \quad\left(\begin{array}{cc}
\lambda_{1} & 1 / 2 \\
1 / 2 & \lambda_{2}
\end{array}\right) \mapsto\left(\begin{array}{cc}
\lambda_{2} & 1 / 2 \\
1 / 2 & \lambda_{1}
\end{array}\right)
\end{aligned}
$$

If we set $\mu=2 \lambda_{1}$ then $s$ and $t$ give the actions $\mu \mapsto \mu+2, \mu \mapsto \frac{\mu}{1-2 \mu}$ and so generate $\Gamma(2)$; conjugation by the final matrix then extends this to $\Gamma(2) \times \Gamma(2)$. Then with

$$
\widetilde{\tau}_{11}^{\prime}=s^{2} t^{-1}\left(\widetilde{\tau}_{11}\right)=-\frac{1}{\mathcal{T}+6}, \quad \widetilde{\tau}_{22}^{\prime}=t\left(\widetilde{\tau}_{22}\right)=\frac{\mathcal{T}+6}{12}
$$

and we have $12 \widetilde{\tau}_{11}^{\prime} \widetilde{\tau}_{22}^{\prime}+1=0$, the relation Bolza's claimed for period matrices for such curves Bol887.

We may now give an alternate characterization of those curves (1.1) satisfying Hitchin's conditions $\mathbf{H 1}$ and $\mathbf{H 2}$.

Proposition 18. The family of curves $\eta^{3}+\chi\left(\zeta^{6}+b \zeta^{3}-1\right)=0$ satisfy the constraints $\mathbf{H 1}$ and $\mathbf{H 2}$ when

$$
b(m, n)=-\frac{\sqrt{3}\left(p(m, n)^{6}-45 p(m, n)^{4}+135 p(m, n)^{2}-27\right)}{9 p(m, n)\left(p(m, n)^{4}-10 p(m, n)^{2}+9\right)}
$$

and $\chi=\chi(m, n)$ may be expressed in terms of $m, n$ and $b(m, n)$ by Proposition 7 . Here $m$ and $n$ are relatively prime integers $(m, n)=1$ for which $(m+n)(m-2 n)<0$ and

$$
p(m, n)=\frac{3 \vartheta_{3}^{2}\left(0 \mid \frac{\mathcal{T}(m, n)}{2}\right)}{\vartheta_{3}^{2}\left(0 \mid \frac{\mathcal{T}(m, n)}{6}\right)}, \quad \mathcal{T}(m, n)=2 \imath \sqrt{3} \frac{n+m}{2 n-m} .
$$

Indeed we may relate the elliptic curves $\mathcal{E}_{ \pm}$of Lemma 9 with the period matrix (4.5) or the symplectically equivalent (4.14) via 
Corollary 19. The genus two curve $\mathcal{C}$ two-sheetedly covers the elliptic curves $\mathcal{E}_{ \pm}$whose Jacobian moduli may be written

$$
k_{+}^{2}=\frac{\vartheta_{2}^{4}\left(0 \mid \frac{\mathcal{T}}{6}\right)}{\vartheta_{3}^{4}\left(0 \mid \frac{\mathcal{T}}{6}\right)}, \quad k_{-}^{2}=\frac{\vartheta_{2}^{4}\left(0 \mid \frac{\mathcal{T}}{2}\right)}{\vartheta_{3}^{4}\left(0 \mid \frac{\mathcal{T}}{2}\right)}
$$

The proof of both the proposition and corollary is presented in Appendix C.

\section{The Theta function Question}

The final step in establishing the existence of monopoles with spectral curve (1.1) is then to understand the vanishing properties of the function

$$
H(y)=h_{-1}(y) h_{0}(y) h_{1}(y)
$$

with the definitions introduced in Proposition 17 $H(y)$ is also an elliptic function with periods $2(n+m) / 3$ and $4(n+m) \rho / 3$. Given the periodicity in $k$ of $h_{k}$ proven in this proposition we have that

Lemma 20. $H(y(\lambda))=0 \Leftrightarrow H(y(\lambda+2))=0$ and these functions have the same vanishing properties.

Numerical calculations in [BE07] suggested the conjecture

Conjecture 21. For each pair of relatively prime integers $(m, n)=1$ for which $(2 n-m)(n+$ $m)>0$ let $y=y(\lambda)=\lambda(n+m) \rho / 3$ and $\mathcal{T}=2 i \sqrt{3}(n+m) /(2 n-m)$. Then $H(y)$ vanishes $2(|n|-1)$ times on the interval $\lambda \in(0,2)$.

To prove the uniqueness of the tetrahedral monopole within the class of symmetric monopole curves it will suffice to show only $(m, n)=(1,1)$ and $(0,1)$ have no zeros within the range. At present we don't know how to prove the more general conjecture. We expect vanishing at $\lambda=0$ and 2 . This follows here due to

Lemma 22. We have the following identities for all $\tau$ in the upper half-plane:

$$
\begin{aligned}
& \frac{\vartheta_{3}\left(\frac{\tau}{3} \mid \tau\right)}{\vartheta_{2}\left(\frac{\tau}{3} \mid \tau\right)}=\frac{\vartheta_{2}\left(\frac{1}{3} \mid \frac{\tau}{3}\right)}{\vartheta_{3}\left(\frac{1}{3} \mid \frac{\tau}{3}\right)} \\
& \vartheta_{4}^{2}(0 \mid \tau) i \sqrt{3} \frac{\vartheta_{1}\left(\frac{\tau}{3} \mid \tau\right) \vartheta_{4}\left(\frac{\tau}{3} \mid \tau\right)}{\vartheta_{2}^{2}\left(\frac{\tau}{3} \mid \tau\right)}+\vartheta_{4}^{2}\left(0 \mid \frac{\tau}{3}\right) \frac{\vartheta_{1}\left(\frac{1}{3} \mid \frac{\tau}{3}\right) \vartheta_{4}\left(\frac{1}{3} \mid \frac{\tau}{3}\right)}{\vartheta_{3}^{2}\left(\frac{1}{3} \mid \frac{\tau}{3}\right)}=0
\end{aligned}
$$

Similar identities may be obtained by cyclic interchange of the $\theta$-subscripts $i, j, k \in\{2,3,4\}$.

As a consequence we obtain

$$
\frac{\vartheta_{3}\left(\frac{\tau}{3} \mid \tau\right)}{\vartheta_{2}\left(\frac{\tau}{3} \mid \tau\right)}=\frac{\vartheta_{3}\left( \pm \frac{\tau}{3} \mid \tau\right)}{\vartheta_{2}\left( \pm \frac{\tau}{3} \mid \tau\right)}=\frac{\vartheta_{3}\left(\frac{2 \tau}{3} \mid \tau\right)}{\vartheta_{2}\left(\frac{2 \tau}{3} \mid \tau\right)}=\frac{\vartheta_{2}\left(\frac{1}{3} \mid \frac{\tau}{3}\right)}{\vartheta_{3}\left(\frac{1}{3} \mid \frac{\tau}{3}\right)}=-\frac{\vartheta_{2}\left(\frac{2}{3} \mid \frac{\tau}{3}\right)}{\vartheta_{3}\left(\frac{2}{3} \mid \frac{\tau}{3}\right)} .
$$

Although we have not seen these identities in the standard texts known to us these identities may be established by standard techniques. We then have,

Lemma 23. At $\lambda=0$ we have

$$
h_{ \pm 1}(0)=0, h_{0}(0) \neq 0,
$$

and each of the functions, $h_{ \pm 1}(y(\lambda))$ vanish to second order in $\lambda$. 
Proof. At the point $y=0$ we have that $h_{ \pm 1}(0)=0$ on account of (5.2). Further the derivatives

$$
\begin{aligned}
& \frac{d}{d z}\left(\frac{\vartheta_{3}}{\vartheta_{2}}(z \mid \mathcal{T})\right)=\pi \vartheta_{4}^{2}(0 \mid \mathcal{T}) \frac{\vartheta_{1}}{\vartheta_{2}}(z \mid \mathcal{T}) \frac{\vartheta_{4}}{\vartheta_{2}}(z \mid \mathcal{T}), \\
& \frac{d}{d z}\left(\frac{\vartheta_{2}}{\vartheta_{3}}(z \mid \mathcal{T})\right)=-\pi \vartheta_{4}^{2}(0 \mid \mathcal{T}) \frac{\vartheta_{1}}{\vartheta_{3}}(z \mid \mathcal{T}) \frac{\vartheta_{4}}{\vartheta_{3}}(z \mid \mathcal{T}) .
\end{aligned}
$$

show that $h_{ \pm 1}^{\prime}(0)=0$ also vanishes on account of (5.3) and so both vanish to second order here. Standard $\theta$-function expansions show that $h_{0}(0)$ is nonvanishing.

A consequence of this lemma is that $H(y(\lambda))$ vanishes to fourth order in $\lambda$ at $\lambda=0$ and 2 ; this is equivalent to the higher order vanishing of $\theta(\lambda \widehat{\boldsymbol{U}} \pm \widetilde{\boldsymbol{K}}, \hat{\tau})$ remarked upon earlier. We may now establish the theorem stated in the introduction.

Proof of Theorem 1. The dependence (4.10) of the modulus $\mathcal{T}=\mathcal{T}(\mathcal{R})$ on the ratio $\mathcal{R}$ means that the vanishing $h_{k}(y)=0$ and $H(y)=0$ define (respectively) implicit functions $y=X_{k}(\mathcal{R})$ and $y=X(\mathcal{R})$ of the real variable $\mathcal{R}$. Although our problem has $\mathcal{R} \in \mathbb{Q}$ we may extend its domain to the whole real half-line, $|\mathcal{R}| \in \mathbb{R}^{+}$(recall our conventions are such that $\mathcal{R}<0)$. The functions $X_{*}(\mathcal{R})$ are clearly multi-valued reflecting the periodicities of $h_{k}(y)$ and $H(y)$. We may determine many points on $X(\mathcal{R})$ using the fundamental Lemma 22. For example, consider $y=2 \rho / 3$ and solutions to $h_{-1}(2 \rho / 3)=0$. Substitution and some simplification leads to solving

$$
\frac{\vartheta_{3}}{\vartheta_{2}}\left(\frac{|\mathcal{R}|+2}{6} \mathcal{T} \mid \mathcal{T}\right)=\frac{\vartheta_{2}}{\vartheta_{3}}\left(\frac{|\mathcal{R}|}{6} \mathcal{T}+\frac{1}{3} \mid \frac{\mathcal{T}}{3}\right) .
$$

Using Lemma 22 we find solutions when

- $|\mathcal{R}|$ is even, giving $|\mathcal{R}|=6 k$ or $6 k+2$ ie $2,6,8,12,14, \ldots$

- $|\mathcal{R}|$ is odd, giving $|\mathcal{R}|=3,5,9,11,15,17, \ldots$..

Similar arguments give solutions

$$
\begin{array}{lccl}
y=2 \rho / 3 & h_{-1} & & |\mathcal{R}|=2,3,5,6,8,9,11,12, \ldots \\
y=2 \rho / 3 & h_{0} & & |\mathcal{R}|=1,2,4,5,7,8,10,11, \ldots \\
y=4 \rho / 3 & h_{0} & & |\mathcal{R}|=1,2,4,5,7,8,10,11 \ldots \\
y=4 \rho / 3 & h_{0} & & |\mathcal{R}|=3 k+1 / 2, \\
y=4 \rho / 3 & h_{1} & & |\mathcal{R}|=2,3,5,6,8,9,11,12, \ldots \\
y=4 \rho / 3 & h_{1} & & |\mathcal{R}|=3 k+1 / 2, \ldots \\
y=2 \rho & h_{1} & & |\mathcal{R}|=1,2,3,4,5,6,7,8,9, \ldots
\end{array}
$$

and so on. At several of these points these points the tangent to $X_{*}(\mathcal{R})$ becomes vertical; these may be obtained by solving the analogous formulae for the tangent. A graph of some of the components of $X(\mathcal{R})$ is given in Figure 2

Now using the symmetry (2.20) we may assume that $n+m \geq 1$ and so for $\lambda \in(0,2)$ then $y / \rho \in(0,2(n+m) / 3) \supseteq(0,2 / 3)$. Now we see that $X(\mathcal{R})$ always has a zero in $(0,2 / 3)$ for all $|\mathcal{R}|=(2 n-m) /(n+m) \in(1,2) \cup(2, \infty)$ and so these values cannot yield a monopole. Similarly if $n+m \geq 2$ there is always a zero of $X(\mathcal{R})$ in $(0,4 / 3)$ for any $|\mathcal{R}| \neq 1 / 2$. Thus we must have either $n+m=1$ and $|\mathcal{R}| \in(0,1] \cup\{2\}$ or $n+m=2$ and $|\mathcal{R}|=1 / 2$. The only solutions to these constraints are $(m, n)=(0,1)$ with $|\mathcal{R}|=2$ and $(m, n)=(1,1)$ with $|\mathcal{R}|=1 / 2$. For all other $(m, n)$ there are solutions to $H(y)=0$ for $\lambda \in(0,2)$ and thus by Proposition 17 they do not yield monopoles. Now the two cases $(m, n)=(0,1),(1,1)$ were 
shown to yield the tetrahedrally symmetric monopoles in BE06. Thus we have established Theorem 1

Remark: We numerically observe that $h_{k}(y)=0 \Longleftrightarrow h_{k}(\rho y)=0 \Longleftrightarrow h_{k}\left(\rho^{2} y\right)=0$. The modulus of the elliptic functions $h_{k}$ is $2 \rho$ so this is not simply complex multiplication. This observation remains unexplained.

\section{Discussion}

This paper has been devoted to the study of certain charge three (centred) $S U(2)$ BPS monopoles. The spectral curve of the general monopole of this class may be put (by a rotation) in the form

$$
\eta^{3}+\eta\left(\alpha_{0} \zeta^{4}+\alpha_{1} \zeta^{3}+\alpha \zeta^{2}-\bar{\alpha}_{1} \zeta+\bar{\alpha}_{0}\right)+\beta \zeta^{6}+\beta_{1} \zeta^{5}+\beta_{2} \zeta^{4}+\gamma \zeta^{3}-\bar{\beta}_{2} \zeta^{2}+\bar{\beta}_{1} \zeta-\beta=0
$$

with $\alpha, \beta$ and $\gamma$ real. Hitchin's constraints on the spectral curve mean there are transcendental relations amongst these coefficients and the outstanding problem is to realise these. Two sorts of problem arise. The first is implementing Hitchin's constraint $\mathbf{H 2}$ coming from the triviality of the line bundle $L^{2}$ on the spectral curve. This leads via the equivalent Ercolani-Sinha constraints (Lemma 2) to requiring the vector of $\mathfrak{b}$-periods of the meromorphic differential $\gamma_{\infty}$ to be a half-period in the period lattice. In BE06 it was shown for the trigonal curve

$$
\eta^{3}+\beta \zeta^{6}+\beta_{1} \zeta^{5}+\beta_{2} \zeta^{4}+\gamma \zeta^{3}-\bar{\beta}_{2} \zeta^{2}+\bar{\beta}_{1} \zeta-\beta=0
$$

how these might be reexpressed in terms of the four a-periods of a specified holomorphic differential. These constraints were then solved for the symmetric curves

$$
\eta^{3}+\beta \zeta^{6}+\gamma \zeta^{3}-\beta=0
$$

and a countable family of curves satisfying this constraint of Hitchin ensued. The problem of requiring a curve with specified periods of a given meromorphic differential arises in many settings within finite-gap integration theory. The bijective correspondence between harmonic maps $T^{2} \rightarrow S^{3}$ and algebro-geometric data, specifying curves with given filling fractions in the AdS/CFT correspondence and seeking closed real geodesics on an ellipsoid all lead to this problem. Finding ways to solve such will be an important area for future research.

The second type of problem and the focus of this paper has been in satisfying Hitchin's constraint H3, the vanishing of a real one parameter family of cohomologies of certain line bundles, $H^{0}\left(\hat{\mathcal{C}}, L^{\lambda}(n-2)\right)=0$ for $\lambda \in(0,2)$. We reexpressed this in terms of the intersection of a real line with the theta divisor $\Theta$ of the curve and the problem is to count the number of intersection points. Again a more general theory is called for. We made progress here utilizing two features of the geometry of our situation. The first was that our curve (1.1) has extra symmetry: it falls within a class studied by Hitchin, Manton and Murray HMM95. when looking at spectral curves of monopoles with spatial symmetries. Curves of the form

$$
\eta^{3}+\alpha \eta \zeta^{2}+\beta \zeta^{6}+\gamma \zeta^{3}-\beta=0
$$

have a cyclic $\mathrm{C}_{3}$ symmetry $(\zeta, \eta) \rightarrow(\rho \zeta, \rho \eta)$ where $\rho^{3}=1$. If $\gamma=0$ this is enlarged to a dihedral symmetry $\mathrm{D}_{3}$ with $(\zeta, \eta) \rightarrow\left(1 / \zeta,-\eta / \zeta^{2}\right)$. Actually the spectral curves themselves have larger symmetry (for any $\gamma$ in the curve (6.1) we have the symmetry $(\zeta, \eta) \rightarrow\left(-1 / \zeta,-\eta / \zeta^{2}\right)$ ) but the nomenclature is based on those symmetries that may be realized as spatial symmetries. This cyclic symmetry means there exists a quotient spectral curve. Here it was of genus 2. We were then able to show that a theorem of Fay and Accola applied and so the problem reduced to one about the theta divisor of the quotient curve (Theorem 14). For a 
cyclically invariant charge $n$ monopole exactly the same considerations apply and we find the genus $(n-1)^{2}$ monopole curve is an $n$-fold unbranched cover of a genus $(n-1)$ hyperelliptic curve. This is the Affine Toda curve of Seiberg-Witten theory observed by Sutcliffe [Sut96. Thus symmetry together with the Fay-Accola theorem reduces the problem significantly. The second simplifying feature reduced the problem to one of elliptic functions: Humbert theory tells us our curve covered an elliptic curve. This feature is a consequence of the great symmetry of our curve and will not persist for the general curves (6.1). Notwithstanding this reduction to questions of elliptic functions we have not proven the general Conjecture 21 counting the number of intersections of the line with the theta divisor. We have however established the uniqueness of the tetrahedrally symmetric monopole within spectral curves of the form (1.1).

An obvious line for further study is to seek those monopoles within the class (6.1). Hitchin, Manton and Murray argued that there were five loci of monopoles within this. These loci are totally geodesic submanifolds of the full moduli space and may be viewed as the orbits of geodesic monopole scattering. Of these loci, one corresponded to $\mathrm{D}_{3}$ symmetric monopoles: asymptotically we have $\alpha^{3}=27 \beta^{2}$ (with $\beta$ large and positive at one end and negative at the other) and half-way along this there is the axisymmetric monopole. The other four loci were isomorphic: at one end asymptotically one has $\alpha^{3}=27 \beta^{2}$ (with $\beta$ of either sign) and $\gamma=0$ while at the other end $\alpha=\pi^{2} / 4-3 b^{2}, \beta=0$ and $\gamma=2 b\left(b^{2}+\pi^{2} / 4\right)$ (with $b$ of either sign). Half-way along this is the tetrahedrally symmetric monopole, the four loci corresponding to four distinct orientations of the tetrahedron. Extending our work to this broader class encounters new difficulties. Although we may use the cyclic symmetry to express the curve and Ercolani-Sinha constraints to ones for the quotient curve, the period integrals arising are not simply expressible in terms of hypergeometric functions and the curve does not cover an elliptic curve. These are significant complications and we hope to pursue this elsewhere.

\section{ACKNOWLEDGEMENTS}

Both authors are grateful to MISGAM for funding a research visit of VZE to Edinburgh in 2009 .

\section{Appendix A. Theta Functions}

For $r \in \mathbb{N}$ the canonical Riemann $\theta$-function is given by

$$
\theta(\boldsymbol{z} \mid \tau)=\sum_{\boldsymbol{n} \in \mathbb{Z}^{r}} \exp \left(\imath \pi \boldsymbol{n}^{T} \tau \boldsymbol{n}+2 \imath \pi \boldsymbol{z}^{T} \boldsymbol{n}\right) .
$$

The $\theta$-function is holomorphic on $\mathbb{C}^{r} \times \mathbb{S}^{r}$ and satisfies

$$
\theta(\boldsymbol{z}+\boldsymbol{p} \mid \tau)=\theta(\boldsymbol{z} \mid \tau), \quad \theta(\boldsymbol{z}+\boldsymbol{p} \tau \mid \tau)=\exp \left\{-\imath \pi\left(\boldsymbol{p}^{T} \tau \boldsymbol{p}+2 \boldsymbol{z}^{T} \boldsymbol{p}\right)\right\} \theta(\boldsymbol{z} \mid \tau),
$$

where $\boldsymbol{p} \in \mathbb{Z}^{r}$.

The Riemann $\theta$-function $\theta_{\boldsymbol{a}, \boldsymbol{b}}(\boldsymbol{z} \mid \tau)$ with characteristics $\boldsymbol{a}, \boldsymbol{b} \in \mathbb{Q}$ is defined by

$$
\begin{aligned}
\theta_{\boldsymbol{a}, \boldsymbol{b}}(\boldsymbol{z} \mid \tau) & \left.=\exp \left\{\imath \pi\left(\boldsymbol{a}^{T} \tau \boldsymbol{a}+2 \boldsymbol{a}^{T}(\boldsymbol{z}+\boldsymbol{b})\right)\right)\right\} \theta(\boldsymbol{z}+\tau \boldsymbol{a}+\boldsymbol{b} \mid \tau) \\
& =\sum_{\boldsymbol{n} \in \mathbb{Z}^{r}} \exp \left\{\imath \pi(\boldsymbol{n}+\boldsymbol{a})^{T} \tau(\boldsymbol{n}+\boldsymbol{a})+2 \imath \pi(\boldsymbol{n}+\boldsymbol{a})^{T}(\boldsymbol{z}+\boldsymbol{b})\right\},
\end{aligned}
$$

where $\boldsymbol{a}, \boldsymbol{b} \in \mathbb{Q}^{r}$. This is also written as

$$
\theta_{\boldsymbol{a}, \boldsymbol{b}}(\boldsymbol{z} \mid \tau)=\theta\left[\begin{array}{l}
\boldsymbol{a} \\
\boldsymbol{b}
\end{array}\right](\boldsymbol{z} \mid \tau) .
$$


For arbitrary $\boldsymbol{a}, \boldsymbol{b} \in \mathbb{Q}^{r}$ and $\boldsymbol{a}^{\prime}, \boldsymbol{b}^{\prime} \in \mathbb{Q}^{r}$ the following formula is valid

$$
\theta_{\boldsymbol{a}, \boldsymbol{b}}\left(\boldsymbol{z}+\boldsymbol{a}^{\prime} \tau+\boldsymbol{b}^{\prime} \mid \tau\right)=\exp \left\{-\imath \pi \boldsymbol{a}^{\prime T} \tau \boldsymbol{a}^{\prime}-2 \imath \pi \boldsymbol{a}^{\prime T} \boldsymbol{z}-2 \imath \pi\left(\boldsymbol{b}+\boldsymbol{b}^{\prime}\right)^{T} \boldsymbol{a}^{\prime}\right\} \times \theta_{\boldsymbol{a}+\boldsymbol{a}^{\prime}, \boldsymbol{b}+\boldsymbol{b}^{\prime}}(\boldsymbol{z} \mid \tau) .
$$
have

The function $\theta_{\boldsymbol{a}, \boldsymbol{b}}(\tau)=\theta_{\boldsymbol{a}, \boldsymbol{b}}(\mathbf{0} \mid \tau)$ is called the $\theta$-constant with characteristic $\boldsymbol{a}, \boldsymbol{b}$. We

$$
\begin{aligned}
& \theta_{-\boldsymbol{a},-\boldsymbol{b}}(\boldsymbol{z} \mid \tau)=\theta_{\boldsymbol{a}, \boldsymbol{b}}(-\boldsymbol{z} \mid \tau) \\
& \theta_{\boldsymbol{a}+\boldsymbol{p}, \boldsymbol{b}+\boldsymbol{q}}(\boldsymbol{z} \mid \tau)=\exp \left(2 \pi \imath \boldsymbol{a}^{T} \boldsymbol{q}\right) \theta_{\boldsymbol{a}, \boldsymbol{b}}(\boldsymbol{z} \mid \tau)
\end{aligned}
$$

The following transformation formula is given in [gu72, p85, p176].

Proposition 24. For any $\mathfrak{g}=\left(\begin{array}{cc}A & B \\ C & D\end{array}\right) \in \operatorname{Sp}(2 g, \mathbb{Z})$ and $(\boldsymbol{a}, \boldsymbol{b}) \in \mathbb{Q}^{2 g}$ we put

$$
\begin{aligned}
\mathfrak{g} \cdot(\boldsymbol{a}, \boldsymbol{b}) & =(\boldsymbol{a}, \boldsymbol{b}) \mathfrak{g}^{-1}+\frac{1}{2}\left(\operatorname{diag}\left(C D^{T}\right), \operatorname{diag}\left(A B^{T}\right)\right) \\
\boldsymbol{\phi}_{\boldsymbol{a}, \boldsymbol{b}}(\mathfrak{g}) & =-\frac{1}{2}\left(\boldsymbol{a} D^{T} B \boldsymbol{a}^{T}-2 \boldsymbol{a} B^{T} C \boldsymbol{b}^{T}+\boldsymbol{b} C^{T} A \boldsymbol{b}^{T}\right)+\frac{1}{2}\left(\boldsymbol{a} D^{T}-\boldsymbol{b} C^{T}\right)^{T} \operatorname{diag}\left(A B^{T}\right),
\end{aligned}
$$

where $\operatorname{diag}(A)$ is the row vector consisting of the diagonal components of $A$. Then for every $\mathfrak{g} \in \operatorname{Sp}(2 g, \mathbb{Z})$ we have

$$
\theta_{\mathfrak{g} \cdot(\boldsymbol{a}, \boldsymbol{b})}\left(0 \mid\left(A \tau_{\mathfrak{b}}+B\right)\left(C \tau_{\mathfrak{b}}+D\right)^{-1}\right)=\kappa(\mathfrak{g}) \exp \left(2 \pi \imath \phi_{\boldsymbol{a}, \boldsymbol{b}}(\mathfrak{g})\right) \operatorname{det}\left(C \tau_{\mathfrak{b}}+D\right)^{\frac{1}{2}} \theta_{(\boldsymbol{a}, \boldsymbol{b})}\left(0 \mid \tau_{\mathfrak{b}}\right)
$$

in which $\kappa(\mathfrak{g})^{2}$ is a 4 -th root of unity depending only on $\mathfrak{g}$ while

$$
\begin{aligned}
\theta_{\mathfrak{g} \cdot(\boldsymbol{a}, \boldsymbol{b})}\left(z\left(C \tau_{\mathfrak{b}}+D\right)^{-1} \mid\left(A \tau_{\mathfrak{b}}+B\right)\left(C \tau_{\mathfrak{b}}+D\right)^{-1}\right)=\mu & \exp \left(i \pi z\left(C \tau_{\mathfrak{b}}+D\right)^{-1} C z^{T}\right) \operatorname{det}\left(C \tau_{\mathfrak{b}}+D\right)^{\frac{1}{2}} \\
& \times \theta_{(\boldsymbol{a}, \boldsymbol{b})}\left(z \mid \tau_{\mathfrak{b}}\right)
\end{aligned}
$$

and $\mu$ is a complex number independent of $\tau$ and $z$ such that $|\mu|=1$.

\section{Appendix B. Proof of Proposition 17}

The proposition follows from Theorem 14. Let us first simplify (4.9) first using (4.11). Using the standard transformation properties,

$$
\begin{aligned}
& \vartheta_{3}\left(z_{1} \mid 1-\frac{1}{\mathcal{T}-2}\right)=\vartheta_{4}\left(z_{1} \mid-\frac{1}{\mathcal{T}-2}\right) \\
& =(-i[\mathcal{T}-2])^{1 / 2} \exp \left[i \pi z_{1}^{2}(\mathcal{T}-2)\right] \vartheta_{2}\left([\mathcal{T}-2] z_{1} \mid \mathcal{T}-2\right) \\
& =(-i[\mathcal{T}-2])^{1 / 2} \exp \left[i \pi z_{1}^{2}(\mathcal{T}-2)-i \pi / 2\right] \vartheta_{2}\left([\mathcal{T}-2] z_{1} \mid \mathcal{T}\right) \\
& \vartheta_{3}\left(z_{1}+1 / 2 \mid 1-\frac{1}{\mathcal{T}-2}\right)=\vartheta_{4}\left(z_{1}+1 / 2 \mid-\frac{1}{\mathcal{T}-2}\right) \\
& =(-i[\mathcal{T}-2])^{1 / 2} \exp \left[i \pi\left(z_{1}+1 / 2\right)^{2}(\mathcal{T}-2)\right] \vartheta_{2}\left([\mathcal{T}-2]\left(z_{1}+1 / 2\right) \mid \mathcal{T}-2\right) \\
& =(-i[\mathcal{T}-2])^{1 / 2} \exp \left[i \pi z_{1}^{2}(\mathcal{T}-2)\right] \vartheta_{3}\left([\mathcal{T}-2] z_{1} \mid \mathcal{T}\right) \\
& \vartheta_{3}\left(2 z_{2} \mid 4 \widetilde{\tau}_{2,2}\right)=\vartheta_{3}\left(2 z_{2} \mid \frac{\mathcal{T}}{3}-2\right)=\vartheta_{3}\left(2 z_{2} \mid \frac{\mathcal{T}}{3}\right)
\end{aligned}
$$




$$
\vartheta_{2}\left(2 z_{2} \mid 4 \widetilde{\tau}_{2,2}\right)=\vartheta_{2}\left(2 z_{2} \mid \frac{\mathcal{T}}{3}-2\right)=\exp (-i \pi / 2) \vartheta_{2}\left(2 z_{2} \mid \frac{\mathcal{T}}{3}\right) .
$$

we obtain

$$
\begin{aligned}
\theta\left(z_{1}, z_{2} ; \widetilde{\mathcal{T}}\right) & =(-i[\mathcal{T}-2])^{1 / 2} \exp \left[i \pi z_{1}^{2}(\mathcal{T}-2)-i \pi / 2\right] \\
& \times\left[\vartheta_{2}\left([\mathcal{T}-2] z_{1} \mid \mathcal{T}\right) \vartheta_{3}\left(2 z_{2} \mid \frac{\mathcal{T}}{3}\right)+\vartheta_{3}\left([\mathcal{T}-2] z_{1} \mid \mathcal{T}\right) \vartheta_{2}\left(2 z_{2} \mid \frac{\mathcal{T}}{3}\right)\right]
\end{aligned}
$$

Now the argument $\boldsymbol{z}=\lambda \boldsymbol{U}^{\prime}+\boldsymbol{K}^{\prime}+k \boldsymbol{l}^{\prime}$ is simplified upon setting

This yields

$$
y=\frac{n+m}{3} \rho \lambda=\frac{n+m}{3} \frac{(-1+i \sqrt{3})}{2} \lambda .
$$

$$
[\mathcal{T}-2] z_{1}=-i \sqrt{3} y+\frac{4}{3} \mathcal{T}-3+k-k \frac{\mathcal{T}}{3}, \quad 2 z_{2}=y+\frac{\mathcal{T}}{6}+\frac{k-1}{3} .
$$

With $A_{\tau}(v)=e^{-i \pi(2 v+\tau)}, B_{\tau}(v)=e^{-i \pi(v+\tau / 4)}$ we have

$$
\begin{aligned}
\vartheta_{2}\left([\mathcal{T}-2] z_{1} \mid \mathcal{T}\right) & =(-1)^{k-1} A_{\mathcal{T}}\left(-\left[i \sqrt{3} y+(k-1) \frac{\mathcal{T}}{3}\right]\right) \vartheta_{2}\left(i \sqrt{3} y+(k-1) \frac{\mathcal{T}}{3} \mid \mathcal{T}\right) \\
\vartheta_{3}\left(2 z_{2} \mid \frac{\mathcal{T}}{3}\right) & =B_{\mathcal{T} / 3}\left(y+\frac{k-1}{3}\right) \vartheta_{2}\left(y+\frac{k-1}{3} \mid \frac{\mathcal{T}}{3}\right) \\
\vartheta_{3}\left([\mathcal{T}-2] z_{1} \mid \mathcal{T}\right) & =A_{\mathcal{T}}\left(-\left[i \sqrt{3} y+(k-1) \frac{\mathcal{T}}{3}\right]\right) \vartheta_{3}\left(i \sqrt{3} y+(k-1) \frac{\mathcal{T}}{3} \mid \mathcal{T}\right) \\
\vartheta_{2}\left(2 z_{2} \mid \frac{\mathcal{T}}{3}\right) & =B_{\mathcal{T} / 3}\left(y+\frac{k-1}{3}\right) \vartheta_{3}\left(y+\frac{k-1}{3} \mid \frac{\mathcal{T}}{3}\right)
\end{aligned}
$$

and substituting these in (B.1) gives us, up to an exponential factor, the function $H_{k-1}(y)$ where we define the functions

$$
\begin{aligned}
H_{k}(y) & :=\vartheta_{3}\left(i \sqrt{3} y+\frac{k \mathcal{T}}{3} \mid \mathcal{T}\right) \vartheta_{3}\left(y+\frac{k}{3} \mid \frac{\mathcal{T}}{3}\right)+(-1)^{k} \vartheta_{2}\left(i \sqrt{3} y+\frac{k \mathcal{T}}{3} \mid \mathcal{T}\right) \vartheta_{2}\left(y+\frac{k}{3} \mid \frac{\mathcal{T}}{3}\right), \\
h_{k}(y): & =\frac{H_{k}(y)}{\vartheta_{2}\left(i \sqrt{3} y+\frac{k \mathcal{T}}{3} \mid \mathcal{T}\right) \vartheta_{3}\left(y+\frac{k}{3} \mid \frac{\mathcal{T}}{3}\right)} \\
& =h_{k}^{I}(y)+(-1)^{k} h_{k}^{I I}(y)=\frac{\vartheta_{3}}{\vartheta_{2}}\left(i \sqrt{3} y+\frac{k \mathcal{T}}{3} \mid \mathcal{T}\right)+(-1)^{k} \frac{\vartheta_{2}}{\vartheta_{3}}\left(y+\frac{k}{3} \mid \frac{\mathcal{T}}{3}\right),
\end{aligned}
$$

where $k \in \mathbb{Z}$ and $\frac{\vartheta_{3}}{\vartheta_{2}}(z \mid \mathcal{T})$ is shorthand for $\frac{\vartheta_{3}(z \mid \mathcal{T})}{\vartheta_{3}(z \mid \mathcal{T})}$. Thus we have shown

$$
f_{k}(\lambda)=0 \Longleftrightarrow H_{k-1}(\lambda)=0
$$

To establish the proposition we observe that the zeros of $H_{k}(y)$ are different from those of $\vartheta_{2}\left(y+\frac{k}{3} \mid \frac{\mathcal{T}}{3}\right)$ and $\vartheta_{3}\left(i \sqrt{3} y+\frac{k \mathcal{T}}{3} \mid \mathcal{T}\right)$. Upon writing $y=w(-1+i \sqrt{3})$ with $w$ real a zero of $\vartheta_{2}\left(y+\frac{k}{3} \mid \frac{\mathcal{T}}{3}\right)$ takes the form

$$
y=w(-1+i \sqrt{3})=m_{1}+\frac{1}{2}-\frac{k}{3}+n_{1} \frac{\mathcal{T}}{3}, \quad n_{1}, m_{1} \in \mathbb{Z}
$$

while a zero of the latter has the form

$$
i \sqrt{3} y=w(-i \sqrt{3}-3)=m_{2}+\frac{1}{2}+\left(n_{2}+\frac{1}{2}-\frac{k}{3}\right) \mathcal{T}, \quad n_{2}, m_{2} \in \mathbb{Z} .
$$


If these were to vanish simultaneously then we see from their imaginary parts that

$$
0=n_{2}+\frac{n_{1}}{3}+\frac{1}{2}-\frac{k}{3}
$$

which is not possible. Hence the zero's of $H_{k}(y)$ are different from those of $\vartheta_{2}\left(y+\frac{k}{3} \mid \frac{\mathcal{T}}{3}\right)$ and $\vartheta_{3}\left(i \sqrt{3} y+\frac{k \mathcal{T}}{3} \mid \mathcal{T}\right)$. Therefore

$$
f_{k}(\lambda)=0 \Longleftrightarrow H_{k-1}(y(\lambda))=0 \Longleftrightarrow h_{k-1}(y(\lambda))=0
$$

and (4.12) is established up making use of Theorem 14

The first periodicity of (4.13) is follows immediately from the periodicity of the theta functions. For the second we note

$$
\begin{aligned}
h_{k}^{I}\left(y+2 \frac{(n+m)}{3}\right) & \left.=\frac{\vartheta_{3}}{\vartheta_{2}}\left(i \sqrt{3} y+\frac{(2 n-m)}{3} \mathcal{T}+\frac{k \mathcal{T}}{3}\right) \mid \mathcal{T}\right)=h_{k-[n+m]}^{I}(y), \\
h_{k}^{I I}\left(y+2 \frac{(n+m)}{3}\right) & =(-1)^{n+m} h_{k-[n+m]}^{I I}(y), \\
h_{k}\left(y+2 \frac{(n+m)}{3}\right) & =h_{k-[n+m]}(y), \\
h_{k}^{I I}\left(y+2 \frac{(n+m)}{3} \rho\right) & \left.=\frac{\vartheta_{3}}{\vartheta_{2}}\left(i \sqrt{3} y-\frac{(n+m)}{3} i \sqrt{3}-(n+m)+\frac{k \mathcal{T}}{3}\right) \mid \mathcal{T}\right) \\
& \left.=(-1)^{n+m} \frac{\vartheta_{3}}{\vartheta_{2}}\left(i \sqrt{3} y-\frac{(2 n-m)}{6} \mathcal{T}+\frac{k \mathcal{T}}{3}\right) \mid \mathcal{T}\right) \\
& =(-1)^{n+m}\left[h_{k-[n+m]}^{I}(y)\right]^{\epsilon(m)} \\
h_{k}^{I I}\left(y+2 \frac{(n+m)}{3} \rho\right) & =\frac{\vartheta_{2}}{\vartheta_{3}}\left(y+\frac{k-[n+m]}{3}+\frac{(2 n-m)}{6} \mathcal{T} \mid \frac{\mathcal{T}}{3}\right)=\left[h_{k-[n+m]}^{I I}(y)\right]^{\epsilon(m)} \\
h_{k}\left(y+2 \frac{(n+m)}{3} \rho\right) & =\left\{\begin{array}{l}
(-1)^{n+m} h_{k-[n+m]}(y) \quad \text { if } m \text { even, } \\
(-1)^{k} g_{k-[n+m]}(y) \quad \text { if } m \text { odd, }
\end{array}\right.
\end{aligned}
$$

where $\epsilon(m)=1$ if $m$ is even and -1 if $m$ is odd and

$$
g_{k}(y):=h_{k}\left(y+\frac{\mathcal{T}}{2}\right)=\frac{H_{k}(y)}{\vartheta_{3}\left(i \sqrt{3} y+\frac{k \mathcal{T}}{3} \mid \mathcal{T}\right) \vartheta_{2}\left(y+\frac{k}{3} \mid \frac{\mathcal{T}}{3}\right)}
$$

Now $y(\lambda+2)=y(\lambda)+2(n+m) \rho / 3$ and the result follows as $h_{k}(y)=0 \Longleftrightarrow g_{k}(y)=0$.

\section{Appendix C. Proof of Proposition 18}

The proof involves three steps. First let us parameterize $M=(2 \imath-b)^{\frac{1}{3}} /\left(b^{2}+4\right)^{\frac{1}{6}}$ of Lemma 9 by

$$
M=\frac{p+\imath \sqrt{3}}{p-\imath \sqrt{3}} .
$$

Then solving for $b$ we obtain the form (4.15),

$$
b=-\frac{\sqrt{3}\left(p^{6}-45 p^{4}+135 p^{2}-27\right)}{9 p\left(p^{4}-10 p^{2}+9\right)} .
$$


The same substitution (C.1) in the Jacobian moduli (2.23) leads to their parametrization

$$
k_{+}^{2}=\frac{(p+1)^{3}(3-p)}{16 p}, \quad k_{-}^{2}=\frac{(p+1)(3-p)^{3}}{16 p^{3}} .
$$

This is the parametrization used by Ramanujan in his hypergeometric relations of signature 3 , see e.g. [BBG95]. The $\theta$-functional representation of the moduli $k_{ \pm}$can be found in [Law89, Section 9.7],

$$
k_{+}=\frac{\vartheta_{2}^{2}(0 \mid \tau)}{\vartheta_{3}^{2}(0 \mid \tau)}, \quad k_{-}=\frac{\vartheta_{2}^{2}(0 \mid 3 \tau)}{\vartheta_{3}^{2}(0 \mid 3 \tau)}, \quad p=\frac{3 \vartheta_{3}^{2}(0 \mid 3 \tau)}{\vartheta_{3}^{2}(0 \mid \tau)} .
$$

To establish the proposition we must show that the parameter $\tau=\mathcal{T}(m, n) / 6$. To do this we next consider a genus two curve with period system $\tau_{11}, \frac{1}{2}, \tau_{22}$. Bolza [Bol886, p.451] showed that the associated genus two curve may be presented in the form

$$
w^{2}=\left(1+z^{2}\right)\left(c^{2}+e^{\prime 2} z^{2}\right)\left(c^{\prime 2}+e^{2} z^{2}\right)
$$

with $c=\theta_{2}^{2} / \theta_{3}^{2}, c^{\prime}=\theta_{4}^{2} / \theta_{3}^{2}, \quad e=\Theta_{2}^{2} / \Theta_{3}^{2}, \quad e^{\prime}=\Theta_{4}^{2} / \Theta_{3}^{2}$ and $\theta_{j}=\vartheta_{j}\left(0 \mid 2 \tau_{11}\right), \quad \Theta_{j}=$ $\vartheta_{j}\left(0 \mid 2 \tau_{22}\right), j=2,3,4$. For the moment let us assume this is our curve $\mathcal{C}$. Both the (unnormalized) holomorphic differentials $z \mathrm{~d} z / w$ and $\mathrm{d} z / w$ reduce to the holomorphic differentials of elliptic curves that we shall call (and shortly identify with) $\mathcal{E}_{ \pm}$by the (respective) substitutions $z^{2}=t$ and $z^{2}=1 / t$,

$$
\frac{1}{2} \frac{\mathrm{d} t}{\sqrt{(1+t)\left(c^{2}+e^{\prime 2} t\right)\left({c^{\prime}}^{2}+e^{2} t\right)}}, \quad-\frac{1}{2} \frac{\mathrm{d} t}{\sqrt{(1+t)\left(c^{2} t+{e^{\prime 2}}^{2}\right)\left({c^{\prime 2}}^{2} t+e^{2}\right)}} .
$$

The Jacobian moduli of $\mathcal{E}_{ \pm}$are then

$$
k_{+}^{2}=\frac{e^{2}}{e^{\prime 2}} \frac{c^{2}-e^{\prime 2}}{c^{\prime 2}-e^{2}}, \quad k_{-}^{2}=\frac{c^{\prime 2}}{c^{2}} \frac{c^{2}-e^{\prime 2}}{c^{\prime 2}-e^{2}} .
$$

Taking the period system (4.14), i.e. $\tau_{11}=-1 /(\mathcal{T}+6), \tau_{22}=(\mathcal{T}+6) / 12$, one can see that

$$
c=\frac{1}{C^{\prime}}, c^{\prime}=\imath \frac{C}{C^{\prime}}, e=-\imath \frac{E}{E^{\prime}}, e^{\prime}=\frac{1}{E^{\prime}}
$$

where

$$
C=\frac{\vartheta_{2}^{2}}{\vartheta_{3}^{2}}\left(0 \mid \frac{\mathcal{T}}{6}\right), C^{\prime}=\frac{\vartheta_{4}^{2}}{\vartheta_{3}^{2}}\left(0 \mid \frac{\mathcal{T}}{6}\right), E=\frac{\vartheta_{2}^{2}}{\vartheta_{3}^{2}}\left(0 \mid \frac{\mathcal{T}}{2}\right), E^{\prime}=\frac{\vartheta_{4}^{2}}{\vartheta_{3}^{2}}\left(0 \mid \frac{\mathcal{T}}{2}\right)
$$

Again using the parametrization (C.3, C.4) we obtain from (C.7) after simplification,

$$
k_{+}^{2}=C^{2}=\frac{\vartheta_{2}^{4}}{\vartheta_{3}^{4}}\left(0 \mid \frac{\mathcal{T}}{6}\right), \quad k_{-}^{2}=E^{2}=\frac{\vartheta_{2}^{4}}{\vartheta_{3}^{4}}\left(0 \mid \frac{\mathcal{T}}{2}\right) .
$$

This has proven $\tau=\mathcal{T}(m, n) / 6$ provided we can establish the curve (C.5) is $\mathcal{C}$ and identify the elliptic curves $\mathcal{E}_{ \pm}$as above.

Our final step then is to show the curve (C.5) is birationally equivalent to the curve (2.21). Indeed let

$$
T=\frac{L+\mu}{L-\mu}, S=\frac{8 \nu}{(L-\mu)^{3}}, \quad \mu=L \frac{T-1}{T+1}, \nu=\frac{L^{3} S}{(T+1)^{3}} .
$$

Then (2.21) transforms to

$$
S^{2}=(T-1)^{6}+2 \frac{b}{L^{3}}\left(T^{2}-1\right)^{3}+(T+1)^{6} .
$$


This curve is of the same form as (C.5) up to scaling of $S$ and $T$. Using the substitution (C.2) this becomes

$$
\begin{aligned}
\left(\frac{S}{\sqrt{2\left(1-b / L^{3}\right)}}\right)^{2}= & 27 \frac{(p-1)^{2}(p+1)^{2}}{p^{2}(p+3)^{2}(-3+p)^{2}} T^{6}+9 \frac{45 p^{2}+18+p^{6}}{p^{2}(p+3)^{2}(-3+p)^{2}} T^{4} \\
& +3 \frac{2 p^{6}+45 p^{4}+81}{p^{2}(p+3)^{2}(-3+p)^{2}} T^{2}+1
\end{aligned}
$$

whereas (C.5) may be written

$$
\begin{aligned}
\left(\frac{w}{c c^{\prime}}\right)^{2}= & \frac{(p+3)^{4}(p+1)^{2}}{(p-1)^{4} p^{2}(-3+p)^{2}} z^{6}+\frac{\left(45 p^{2}+18+p^{6}\right)(p+3)^{2}}{(p-1)^{4} p^{2}(-3+p)^{2}} z^{4} \\
& +\frac{2 p^{6}+45 p^{4}+81}{p^{2}(-3+p)^{2}(p-1)^{2}} z^{2}+1 .
\end{aligned}
$$

These coincide with $z=\sqrt{3}(p-1) T /(p+3)$. The substitution $W=T^{2}$ reduces the canonical differentials $d T / S$ and $T d T / S$ to the canonical differentials of the elliptic curves $\mathcal{E}_{ \pm}$given in Lemma 9, These correspond to the differentials and curves $\mathcal{E}_{ \pm}$identified above.

In the course of the proof we find the $\theta$-constant representation for the Jacobian moduli of the curves $\mathcal{E}_{ \pm}$,

(C.8) $k_{+}(m, n)=\frac{\vartheta_{2}^{2}\left(0 \mid \frac{\mathcal{T}(m, n)}{6}\right)}{\vartheta_{3}^{2}\left(0 \mid \frac{\mathcal{T}(m, n)}{6}\right)}, \quad k_{-}(m, n)=\frac{\vartheta_{2}^{2}\left(0 \mid \frac{\mathcal{T}(m, n)}{2}\right)}{\vartheta_{3}^{2}\left(0 \mid \frac{\mathcal{T}(m, n)}{2}\right)}, \quad \mathcal{T}(m, n)=2 \imath \sqrt{3} \frac{n+m}{2 n-m}$

which yields the corollary.

\section{REFERENCES}

[Acc71] Robert D. M. Accola, Vanishing Properties of Theta Functions for Abelian Covers of Riemann Surfaces, p7-18 in Advances in the Theory of Riemann Surfaces: Proceedings of the 1969 Sony Brook Conference, edited by L.V. Ahlfors, L. Bers, H.M. Farkas, R.C. Gunning, I. Kra and H.E. Rauch (Princeton University Press 1971).

[BE55] H. Bateman and A. Erdelyi, Higher Transcendental Functions, vol. 2, McGraw-Hill, New York, 1955.

[BBE94] E. D. Belokolos, A. I. Bobenko, V. Z. Enolskii, A. R. Its, and V. B. Matveev, Algebro Geometric Approach to Nonlinear Integrable Equations, Springer, Berlin, 1994.

[BBG95] B. C. Berndt, S. Bhargava, and F. G. Garvan, Rananujans's theories of elliptic functions to alternative bases, Trans. Amer. Math. Soc. 347 (1995), no. 11, 4163-4244.

[Bol886] Oskar Bolza, Ueber die Reduction hyperelliptischer Integrale erster Ordnung und erster Gattung auf elliptische durch eine Transformation vierten Grades, Math.Ann. 28 (1886), 447-456.

[Bol887] Oskar Bolza, On Binary Sextics with Linear Transformations into Themselves, Amer. J. Math. 10 (1887), no. 1, 47-70.

[Ber98] Bruce C. Berndt, Ramanujan's Notebooks Part V, Springer-Verlag, New York, 1998.

[BE06] H. W. Braden and V. Z. Enolski, Remarks on the complex geometry of 3-monopole, arXiv: math-ph/0601040, 2006.

[BE07] Monopoles, Curves and Ramanujan, Reported at Riemann Surfaces, Analytical and Numerical Methods, Max Planck Instititute (Leipzig), 2007. Submitted, arXiv: math-ph/0704.3939.

[BE08] - Finite-gap integration of the SU(2) Bogomolny equation, Glasgow Math. J. (2008), arXiv: math-ph/0806.1807.

[ES89] N. Ercolani and A. Sinha, Monopoles and Baker Functions, Commun. Math. Phys. 125 (1989), $385-416$.

[Fay73] J. D. Fay, Theta functions on Riemann surfaces, Lectures Notes in Mathematics (Berlin), vol. 352, Springer, 1973.

[Hit82] N. J. Hitchin, Monopoles and Geodesics, Commun. Math. Phys. 83 (1982), 579-602. 
[Hit83] - On the Construction of Monopoles, Commun. Math. Phys. 89 (1983), 145-190.

[HMM95] N. J. Hitchin, N. S. Manton and M. K. Murray, Symmetric monopoles, Nonlinearity 8 (1995), 661-692.

[HMR00] C. J. Houghton, N. S. Manton, and N. M. Romão, On the constraints defining BPS monopoles, Commun. Math. Phys. 212 (2000), 219-243. arXiv: hep-th/9909168, 1999.

[Igu72] J. Igusa, Theta Functions, Grund. Math. Wiss., vol. 194, Springer, Berlin, 1972.

[Kra03] A. Krazer, Lehrbuch der Thetafunktionen, Teubner, Leipzig, 1903, reprinted by AMS Chelsea Publishing, 1998.

[MS04] Nicholas Manton and Paul Sutcliffe, Topological Solitons, Cambridge University Press, Cambridge 2004.

[Nah82] W. Nahm, The construction of all self-dual multimonopoles by the ADHM method, in Monopoles in Quantum Field Theory, edited by N.S. Craigie, P. Goddard and W. Nahm (World Scientific, Singapore 1982).

[Mat01] K. Matsumoto, Theta constants associated with the cyclic triple coverings of the complex projective line branching at six points, Publ.Res.Inst.Math.Sci 37 (2001), 419-440, arXiv: math.AG/0008025

[Mur94] N. Murabayashi, The moduli space of genus 2 covering elliptic curve, Manuscripta math. 84 (1994), 125-133.

[Sut96] Paul M. Sutcliffe, Seiberg-Witten theory, monopole spectral curves and affine Toda solitons, Phys. Lett. B381 (1996), 129-136.

[Wel899] J. Wellstein, Zur Theorie der Functionenclasse $s^{3}=\left(z-\alpha_{1}\right)\left(z-\alpha_{2}\right) \ldots\left(z-\alpha_{6}\right)$, Math. Ann. 52 (1899), 440-448.

School of Mathematics, Edinburgh University, Edinburgh.

E-mail address: hwb@ed.ac.uk

Institute of Magnetism, National Academy of Sciences of Ukraine.

E-mail address: vze@ma.hw.ac.uk 

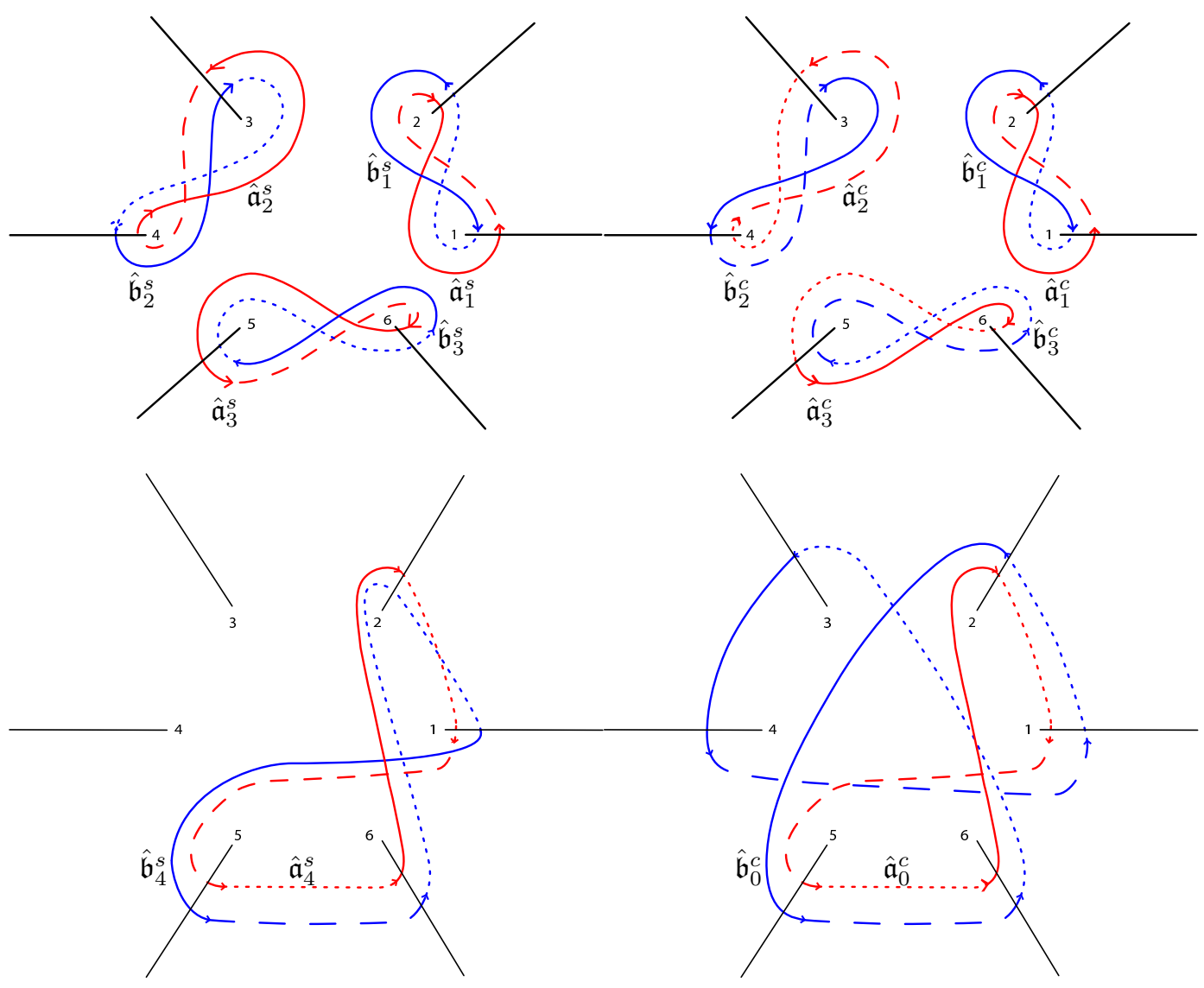

$\begin{array}{ll}\text { (a) The symmetric basis } \hat{\mathfrak{a}}_{*}^{s}, \hat{\mathfrak{b}}_{*}^{s} & \text { (b) The cyclic basis } \hat{\mathfrak{a}}_{*}^{c}, \hat{\mathfrak{b}}_{*}^{c}\end{array}$

Figure 1 . The homology bases. Sheet 1 is denoted by a solid line; sheet 2 by a dashed line; and sheet 3 by a dotted line. 
Figure 2. The plot shows (some) branches of the multi-valued function $y=X(\mathcal{R})$ given implicitly by the equation $H(y)=0$. Circles on the plot shows points at which the tangent lines are vertical. The bold lines correspond to $|\mathcal{R}|=2$ and $1 / 2$. The different colours correspond to branches of $y=X_{k}(\mathcal{R})\left(\right.$ blue $=X_{1}$, green $=X_{0}$, red $\left.=X_{1}\right)$.

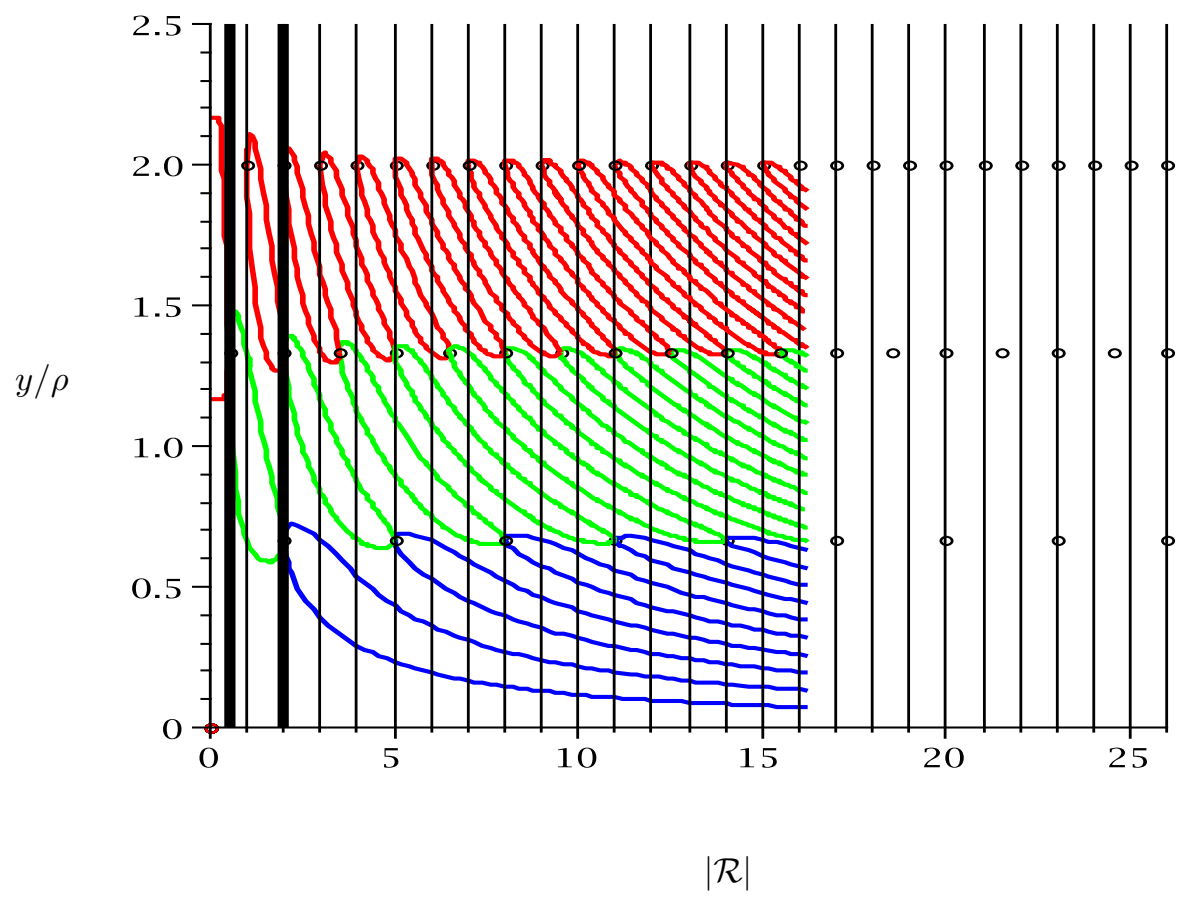

